\title{
Maternal disturbance in activated sphingolipid metabolism causes pregnancy loss in mice
}

\author{
Kiyomi Mizugishi, ${ }^{1}$ Cuiling Li, ${ }^{1}$ Ana Olivera, ${ }^{2}$ Jacek Bielawski, ${ }^{3}$ \\ Alicja Bielawska, ${ }^{3}$ Chu-Xia Deng, ${ }^{1}$ and Richard L. Proia ${ }^{1}$ \\ ${ }^{1}$ Genetics of Development and Disease Branch, NIDDK, and 2Molecular Immunology and Inflammation Branch, \\ National Institute of Arthritis and Musculoskeletal and Skin Diseases, NIH, Bethesda, Maryland, USA. \\ ${ }^{3}$ Department of Biochemistry and Molecular Biology, Medical University of South Carolina, Charleston, South Carolina, USA.
}

\begin{abstract}
Uterine decidualization, a process that occurs in response to embryo implantation, is critical for embryonic survival and thus is a key event for successful pregnancy. Here we show that the sphingolipid metabolic pathway is highly activated in the deciduum during pregnancy and disturbance of the pathway by disruption of sphingosine kinase ( $S p h k$ ) genes causes defective decidualization with severely compromised uterine blood ves-

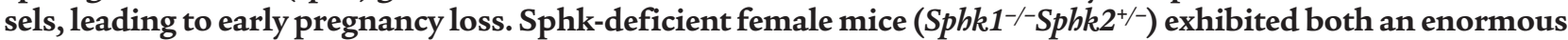
accumulation of dihydrosphingosine and sphingosine and a reduction in phosphatidylethanolamine levels in pregnant uteri. These mice also revealed increased cell death in decidual cells, decreased cell proliferation in undifferentiated stromal cells, and massive breakage of decidual blood vessels, leading to uterine hemorrhage and early embryonic lethality. Thus, sphingolipid metabolism regulates proper uterine decidualization and blood vessel stability. Our findings also suggest that disturbance in sphingolipid metabolism may be considered as a cause of pregnancy loss in humans.
\end{abstract}

\section{Introduction}

Embryonic implantation is a complex series of processes that establishes the connection between maternal and embryonic tissues and requires an intricate program of uterine preparation $(1,2)$. Very soon after implantation, which occurs in the mouse on day 4.5 postcoitum (pc) (day 0.5 - vaginal plug), endometrial stromal cells surrounding implanting blastocysts undergo dramatic transformation (decidualization), during which they proliferate and differentiate into decidual cells. Decidualization begins in the stromal region immediately surrounding the embryo (antimesometrial site). Next to the implanting blastocyst, thin, dense, avascular cell layers, called the primary decidual zone, are formed. Adjacent to the primary decidual zone, the broad secondary decidual zone is fully developed by day $6.5 \mathrm{pc}$, and is characterized by terminally differentiated decidual polyploidy with acquisition of large mono- or binucleated cells (3). The decidua provides a vascular network for nutrition and gas exchange for the developing embryo before a functional placenta is established (4). It also acts as a barrier to uncontrolled trophoblast proliferation.

The sphingolipid metabolic pathway produces bioactive signaling metabolites as well as complex lipids that are utilized in membrane organization and structure. A very prominent signaling lipid is sphingosine-1-phosphate (S1P), which enhances cell survival and growth (5-8). Recent studies have demonstrated its physiologic importance in the development of the vascular and nervous system (9-12), the heart (13), and the immune system (14-16) by signaling through a family of $\mathrm{G}$ protein-coupled receptors designated $\mathrm{S}_{1} \mathrm{P}_{1-5}$. In contrast, 2 precursors of $\mathrm{S} 1 \mathrm{P}$,

Nonstandard abbreviations used: CL, cardiolipin; $\mathrm{E}_{2}$, estrogen; LPA, lysophosphatidic acid; $\mathrm{P}_{4}$, progesterone; $\mathrm{pc}$, postcoitum; $\mathrm{PC}$, phosphatidylcholine; $\mathrm{PE}$, phosphatidylethanolamine; $\mathrm{PGE}_{2}$, prostaglandin $\mathrm{E}_{2}$; PS, phosphatidylserine; ptgs2, PG-endoperoxidase synthase-2; S1P, sphingosine-1-phosphate; Sphk, sphingosine kinase. Conflict of interest: The authors have declared that no conflict of interest exists. Citation for this article: J. Clin. Invest. 117:2993-3006 (2007). doi:10.1172/JCI30674 ceramide and sphingosine, have generally been considered proapoptotic and antigrowth via modulation of key intracellular signaling pathways $(17,18)$. The sphingolipid metabolic pathway also functions to produce membrane lipids by the direct synthesis of complex sphingolipids such as sphingomyelin and glycosphingolipids and by providing precursors for glycerophospholipid synthesis.

Sphingosine kinase (Sphk) is a key enzyme in the sphingolipid metabolic pathway that catalyzes the ATP-dependent phosphorylation of sphingosine to form S1P. Two isoforms of mammalian Sphk (Sphk1 and Sphk2) have been cloned and characterized $(19,20)$. Recently, Sphk1-null and Sphk2-null mice have been created, both of which were phenotypically normal, fertile, and had normal life spans $(12,21,22)$. In contrast, double-knockout Sphk1-/-Sphk2-/mice, which exhibited a deficiency of S1P, were embryonic lethal around E11.5 due to severe defects in neurogenesis and angiogenesis. Taken together, these studies indicate redundant functions of Sphk1 and Sphk2 in mice (12).

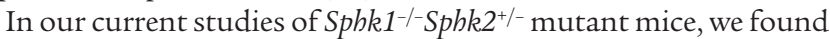

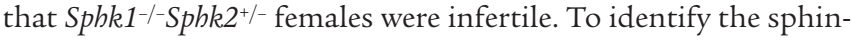
golipid-dependent mechanism affecting female reproduction, we

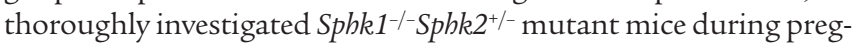
nancy. Here we show that the sphingolipid metabolic pathway is highly activated in the decidua during normal pregnancy and that the disturbance in the activated pathway by disruption of Sphk genes alters levels of key sphingolipid signaling metabolites, causing defects in decidual cells and decidual blood vessels, and leads to early pregnancy loss.

\section{Results}

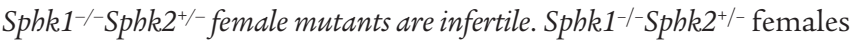
were phenotypically normal. Unexpectedly, however, when they were mated, they were found to be infertile. Mating of Sphk1-/ $S p h k 2^{+/-}$females with fertile males of any genotype did not pro-

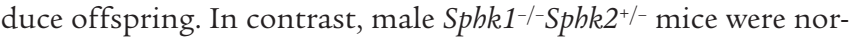


A

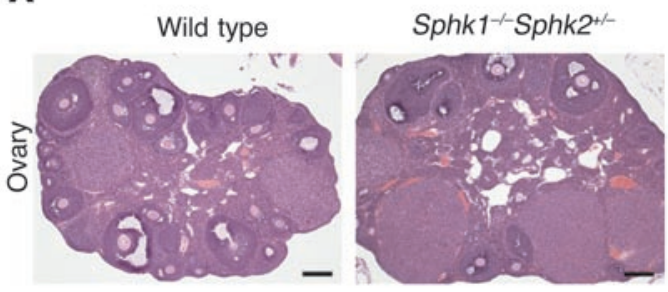

C

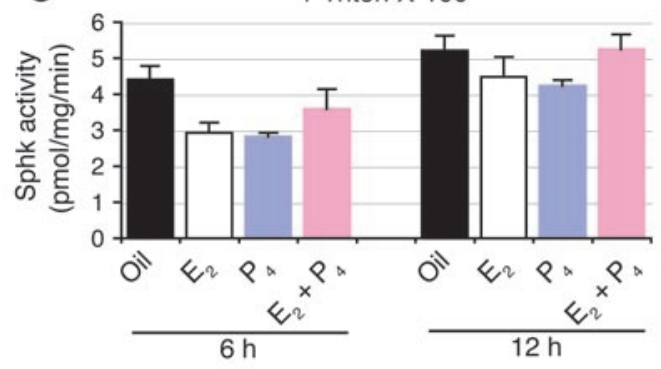

E

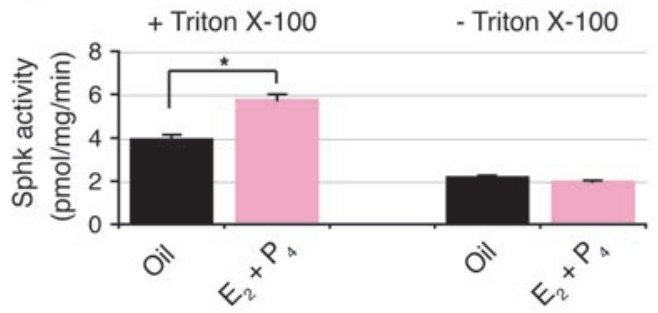

$\mathbf{G}$

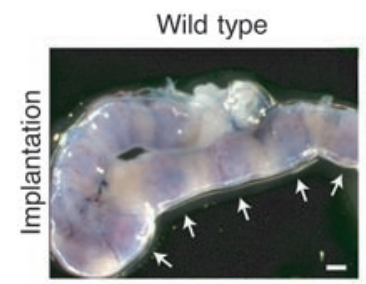

Sphk $1^{--}$Sphk2+-

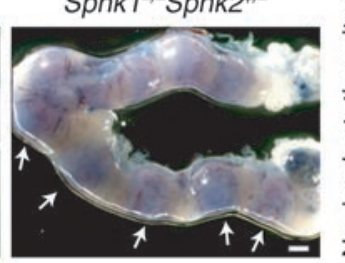

B

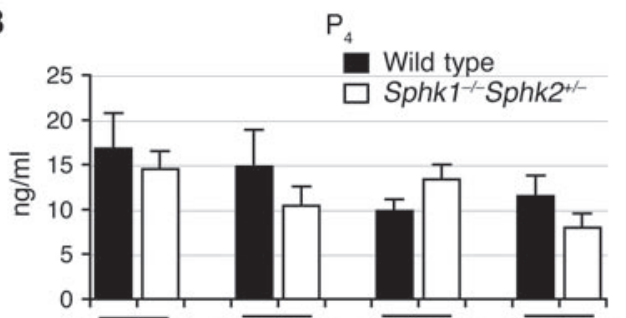

Day $\overline{6.5}$ pc Day 7.5 pc Day 8.5 pc Day 11.5 pc

\section{D}

- Triton X-100
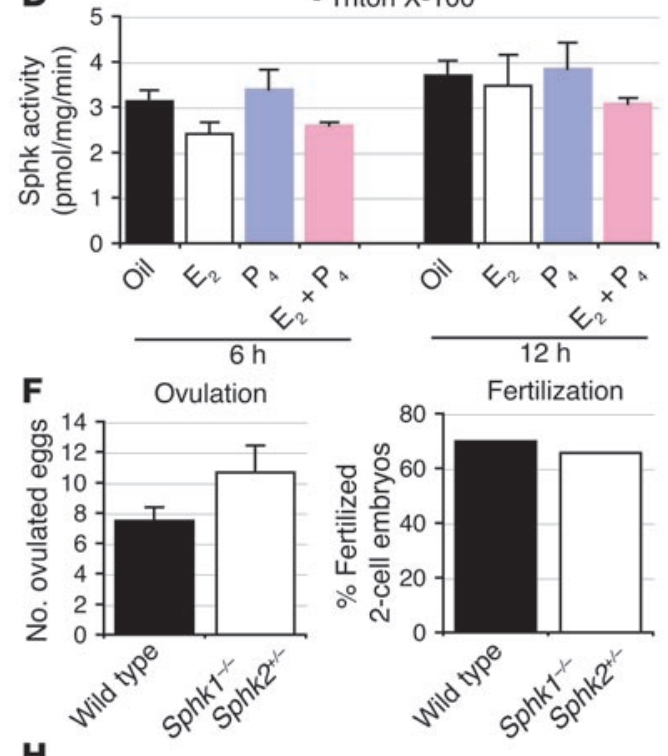

H

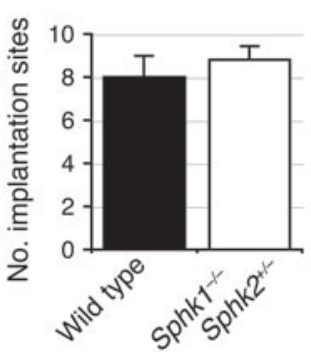

Figure 1

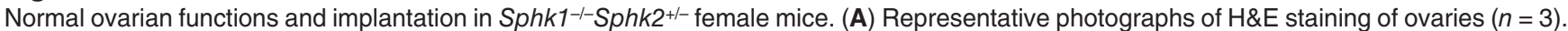
(B) Serum $\mathrm{P}_{4}$ levels on day $6.5 \mathrm{pc}$, day $7.5 \mathrm{pc}$, day $8.5 \mathrm{pc}$, and day $11.5 \mathrm{pc}(n=3)$. (C-E) Measurement of Sphk activity in ovariectomized wildtype females. The mice were given a single injection of $E_{2}\left(100 \mathrm{ng} /\right.$ mouse), a single injection of $P_{4}(2 \mathrm{mg} / \mathrm{mouse})$, or $E_{2}$ plus $P_{4}$, and sacrificed 6 hours or 12 hours later $(\mathbf{C}$ and $\mathbf{D})$. Another group of mice received a regimen designed to mimic the $\mathrm{P}_{4}$ and $\mathrm{E}_{2}$ levels during the estrous cycle and early pregnancy described in Methods (E). The assay was performed in the presence of Triton X-100 (C and E) or of BSA complexes without Triton X-100 (D and E). The data represent mean values \pm SE $\left(n=3,{ }^{*} P<0.01\right.$, paired Student's $t$ test). (F) Ovulation and fertilization rates on day 1.5 pc. Results of ovulation are mean values \pm SE. $n=4$ (wild-type), $n=3$ (Sphk1--Sphk2 ${ }^{+-}$), unpaired Student's $t$ test. (G) Representative photographs of uteri with implantation sites (blue bands) on day 5.5 pc. Arrows indicate implantation sites. (H) The number and weight of implantation sites were examined on day 5.5 pc by the blue dye method. The data represent mean values \pm SE. $n=3$ (wild-type); $n=5$

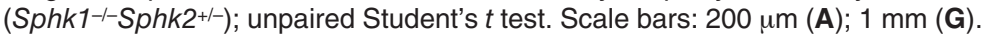

mally fertile. Normal mating behavior was observed in the mutant females when they were caged with males. To determine the causes

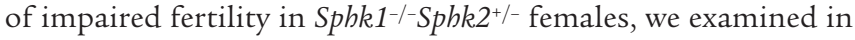
detail the reproductive phenotypes of these mice during pregnancy. The appearance and histology of the ovaries were normal

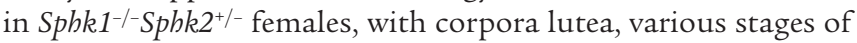
follicular development, and lesser amounts of interstitial tissue, which are all compatible with wild-type mice (Figure 1A).
Circulating serum progesterone $\left(\mathrm{P}_{4}\right)$ levels in Sphk1-/-Sphk2+/females were not significantly different from those in wild-type females on days 6.5 pc, 7.5 pc, 8.5 pc, and 11.5 pc (Figure 1B), suggesting that luteal $\mathrm{P}_{4}$ secretion is normal during pregnancy in the mutant females. Although the $\mathrm{P}_{4}$ levels showed a downward trend as developmental stages advanced, it was not statistically significant. To determine whether Sphk activity is regulated by ovarian steroid hormones, ovariectomized mice received a subcutaneous injection 

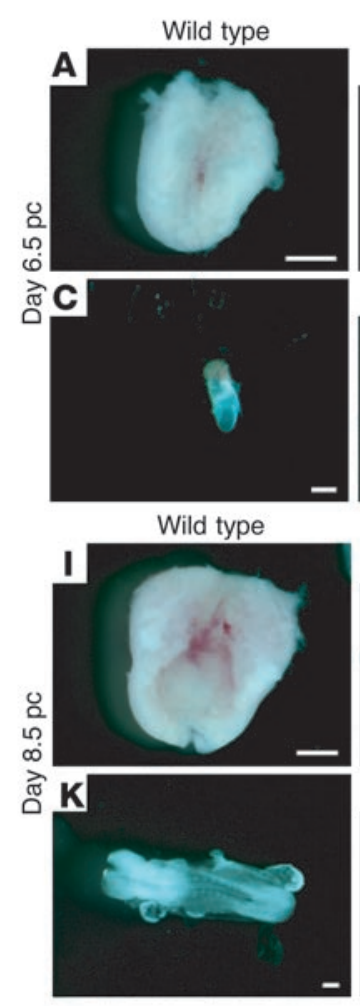

Q

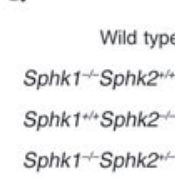

Normal
Sphk1--Sphk2+-

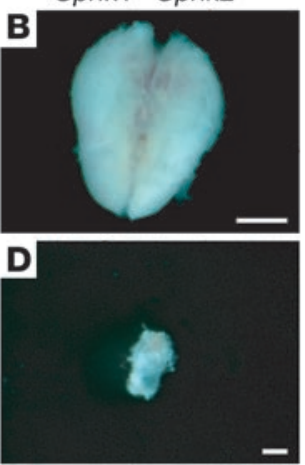

Sphk $1^{-1-}$ Sphk2t/-
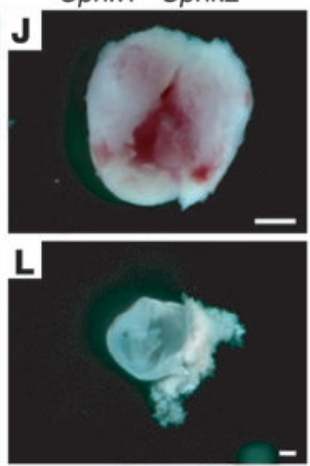

Day 7.5 pc

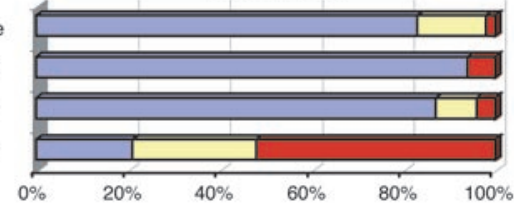

Developmental delay $\square$ Absorbed
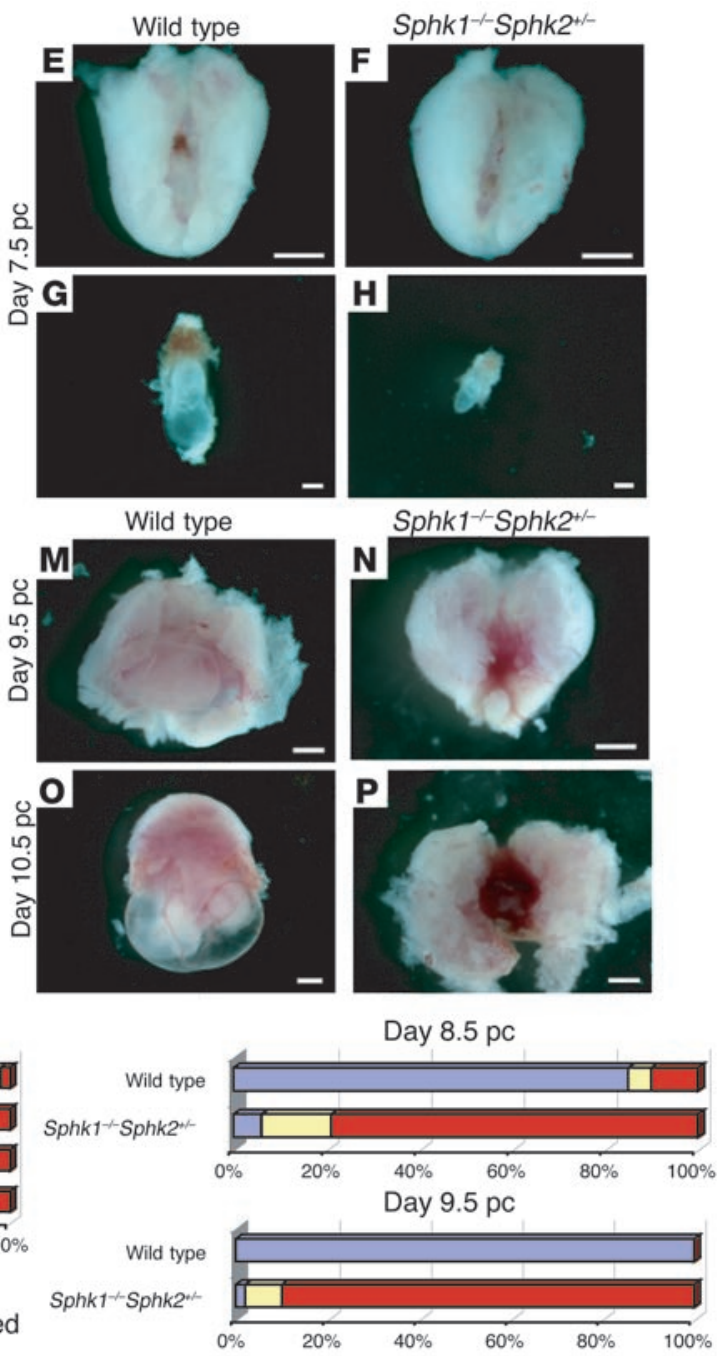

Figure 2

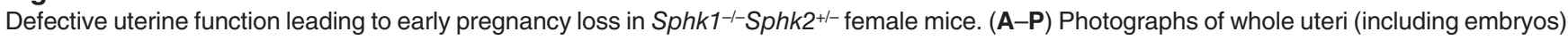
and embryos on day $6.5 \mathrm{pc}(\mathbf{A}-\mathbf{D})$, day $7.5 \mathrm{pc}(\mathbf{E}-\mathbf{H})$, day $8.5 \mathrm{pc}(\mathbf{I}-\mathbf{L})$, day $9.5 \mathrm{pc}(\mathbf{M}$ and $\mathbf{N})$, and day $10.5 \mathrm{pc}(\mathbf{O}$ and $\mathbf{P})$. (A, E, I, M, and $\mathbf{O}) \mathbf{W h o l e}$

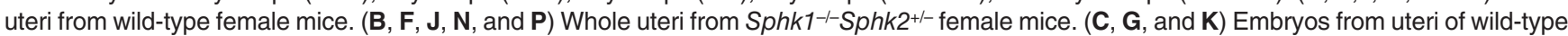

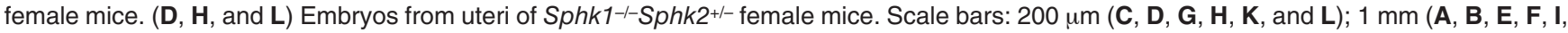
J, and M-P). (Q) Summary of the embryonic phenotype from day $7.5 \mathrm{pc}$ (wild-type, $n=256$; Sphk $1^{-l^{-}}$Sphk2 $2^{+/+}, n=101 ;$ Sphk $1^{+/+}$Sphk2 $2^{-/-}, n=69$;

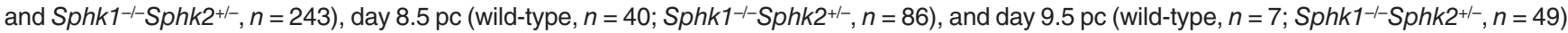
uteri. Embryos were counted according to this classification: normal, developmental delay (the length of long axis: $<1.0 \mathrm{~mm}$, day $7.5 \mathrm{pc} ;<2.0 \mathrm{~mm}$, day $8.5 \mathrm{pc} ;<2.5 \mathrm{~mm}$, day $9.5 \mathrm{pc}$ ), or absorbed, and the percentages were calculated for inclusion in the figure.

of estrogen ( $\mathrm{E}_{2} ; 100 \mathrm{ng} /$ mouse), $\mathrm{P}_{4}\left(2 \mathrm{mg} /\right.$ mouse), or both $\left(\mathrm{E}_{2}+\mathrm{P}_{4}\right)$, and were sacrificed at 6 and 12 hours. Mice injected with oil (vehicle) served as controls. Total Sphk activity was measured in homogenates of whole uteri in the presence of Triton X-100 (Figure 1C), which stimulates Sphk1 and inhibits Sphk2. The Sphk activity was not significantly induced by any injections (Figure 1C). Similar results were obtained when the assay was run in the absence of Triton X-100 and with sphingosine added as a complex with BSA, conditions in which both Sphk1 and Sphk2 are normally active (Figure 1D). When ovariectomized mice were treated with a regimen that mimics the estradiol and $\mathrm{P}_{4}$ levels of the estrous cycle and early pregnancy described in Methods, Sphk activity was slightly increased (1.5-fold) in the presence of Triton X-100, suggesting that the Sphk1 activity could be weakly induced by $\mathrm{P}_{4}$ with estrogen priming (Figure 1E).
Ovulation and fertilization were examined by counting the number of ovulated eggs and fertilized 2-cell embryos on day $1.5 \mathrm{pc}$. There were no significant differences in the ovulated eggs and fertilization

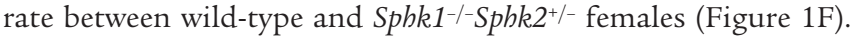
Implantation was assessed by using a blue dye reaction and gross histological analysis. The number, size, weight and intensity of implan-

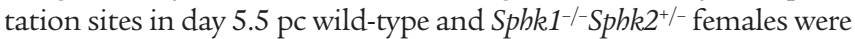
similar (Figure 1, G and $\mathrm{H}$ ), indicating that normal implantation had occurred in the mutant females. There were no spacing abnormalities (Figure 1G). These results suggest that the infertility observed in

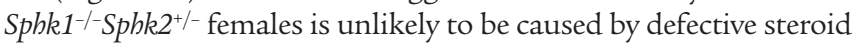
hormone secretion, ovulation, fertilization, or implantation.

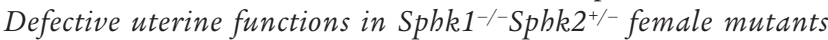
lead to pregnancy loss. To elucidate the cause of the infertility in 


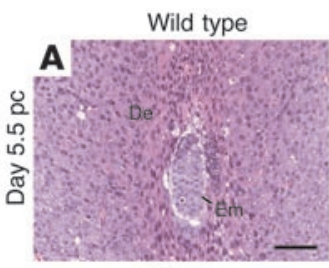

Wild type

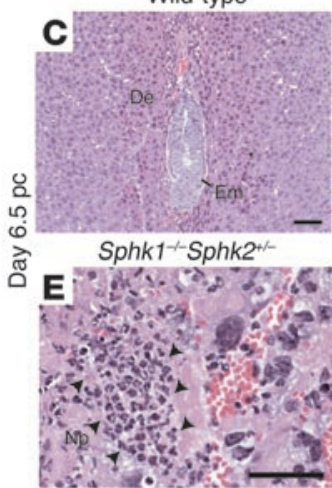

Antisense

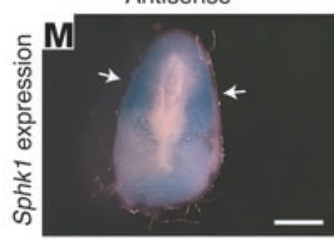

Sphk1+-Sphk2+-

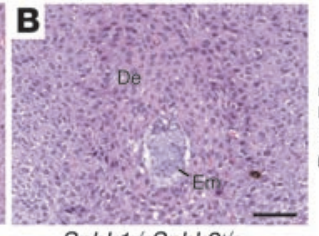

Sphk1-Sphk2

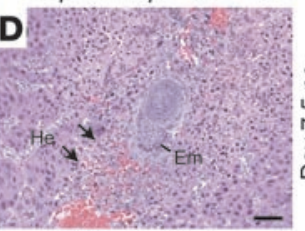

Sphk1--Sphk2

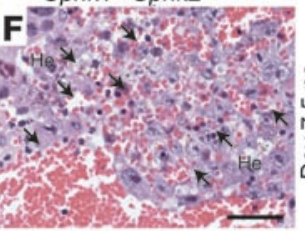

Sense

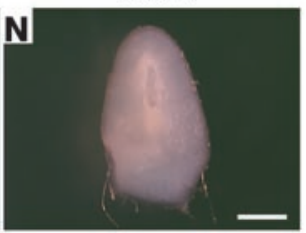

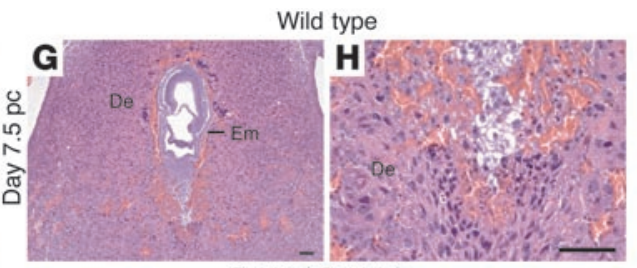

Sphk1-Sphk2
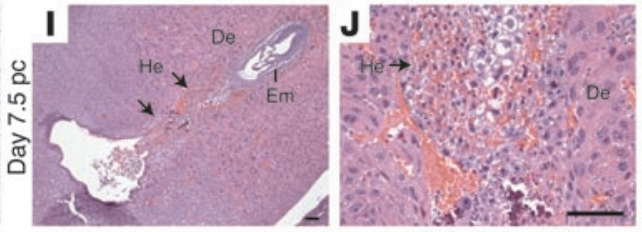

Anti-neutrophil
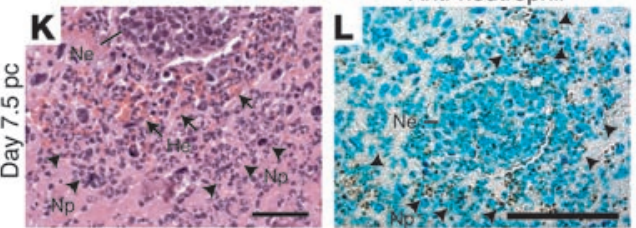

O

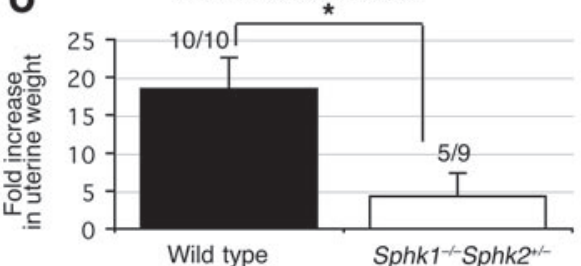

\section{Figure 3}

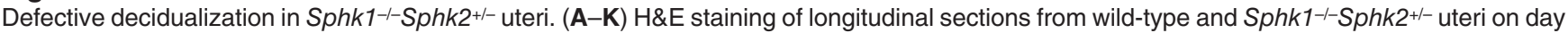

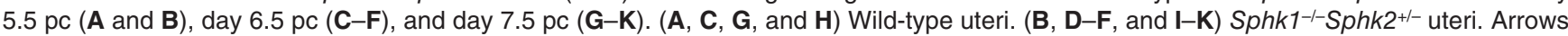
in $\mathbf{D}, \mathbf{F}$, and $\mathbf{I}-\mathbf{K}$ indicate hemorrhage, and arrowheads in $\mathbf{E}$ and $\mathbf{K}$ indicate neutrophil infiltration in the decidua of $S p h k 1^{-/-S p h k 2^{+/-}}$uteri. (L)

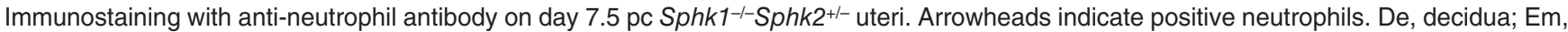
embryo; He, hemorrhage; Np, neutrophil; Ne; necrotic embryo. (M and $\mathbf{N}$ ) In situ hybridization for Sphk1 in wild-type uteri using antisense (M) or sense $(\mathbf{N})$ probes. Arrows in $\mathbf{M}$ indicate Sphk1 expression. Scale bars: $100 \mu \mathrm{m}(\mathbf{A}-\mathbf{D}, \mathbf{G}-\mathbf{J}$, and $\mathbf{L}) ; 50 \mu \mathrm{m}(\mathbf{E}, \mathbf{F}$, and $\mathbf{K}) ;$ and $1 \mathrm{~mm}(\mathbf{M}$ and $\mathbf{N})$. In

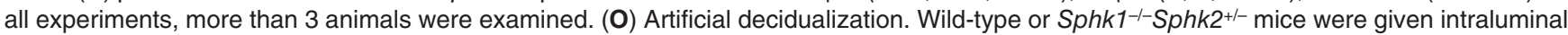
oil infusion on day $3.5 \mathrm{pc}$ of pseudopregnancy. On day $7.5 \mathrm{pc}$, the uteri were weighed. Fold increases denote comparison of weights between infused and noninfused uterine horns. The numbers above the bars indicate the number of responding mice/total number of mice. Results are expressed as mean \pm SE. ${ }^{*} P<0.01$, unpaired Student's $t$ test.

Sphk1 $1^{-/-}$Sphk2 $2^{+/-}$mice, pregnant uteri and embryos were inspected at various developmental stages. In this study, all females were mated with wild-type males. On day $6.5 \mathrm{pc}$, embryos in Sphk1-/-Sphk2+/- uteri did not differ significantly from those in wild-type uteri (Figure 2, A-D). On day 7.5 pc, however, $27 \%$ of embryos examined $(n=243)$ in Sphk1 $1^{-/} S p h k 2^{+/-}$uteri were considerably smaller than those found in wild-type uteri (Figure 2, $\mathrm{E}-\mathrm{H}$ and Q). The embryos were frequently found absorbed even at this stage (52\%) (Figure 2Q). Some embryos (21\%) still looked

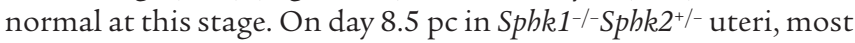
embryos had been absorbed $(79 \%, n=86)$, just leaving traces of yolk sac and no placenta formations (Figure 2, I-L and Q). On

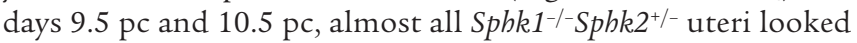
dark red, reminiscent of hemorrhage, in sharp contrast with the normally developed embryos and placentas in wild-type uteri (Figure 2, M-P).

To exclude embryonic effects, day 3.5 pc embryos were collected from wild-type females that had been mated with wild-type males and transferred to the uteri of recipient pseudopregnant (day $2.5 \mathrm{pc}$ )

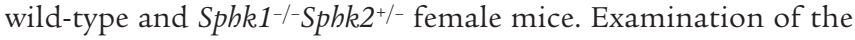

uteri at day 8.5 pc indicated that wild-type embryos were unable

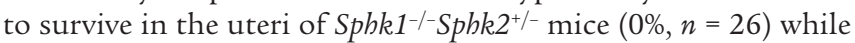
most of the embryos survived in the uteri of wild-type mice (71\%, $n=28)$. Furthermore, there was no difference in the number of pups derived from wild-type $(8.8, n=29)$ and $S p h k 1^{-/-S p h k 2^{+/-}}$(9.0, $n=27$ ) females on day $7.5 \mathrm{pc}$. Taken together, these results dem-

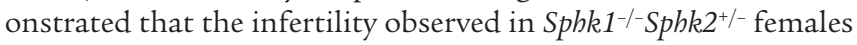
is attributable to maternal effects.

Uterine decidualization is impaired in pregnant Sphk1-/-Sphk2+/- mice. To explore the pathological changes that underlie the pregnancy

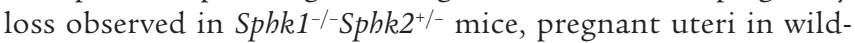

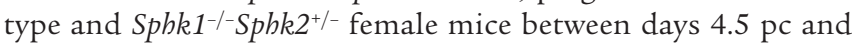
7.5 pc were examined histologically. On day 4.5 and day 5.5 pc,

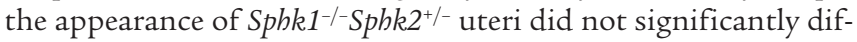
fer from that of uteri from wild-type mice. The decidual cells and embryos in the mutant uteri were morphologically indistinguishable from those in wild-type, suggesting that the decidualization had begun normally in the mutant mice (Figure 3, A and B, and data not shown). However, day 6.5 pc uteri from Sphk1-/-Sphk2+/mice revealed hemorrhage and multinucleated neutrophil infiltra- 


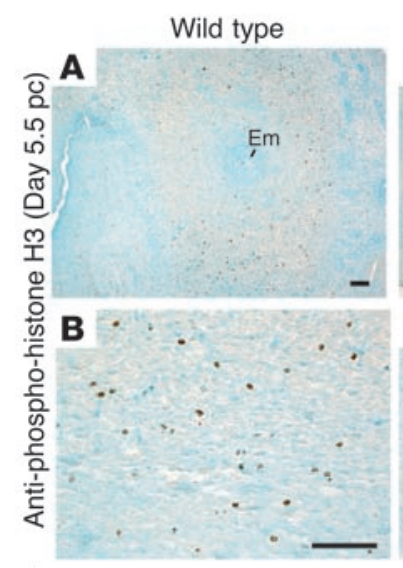

(

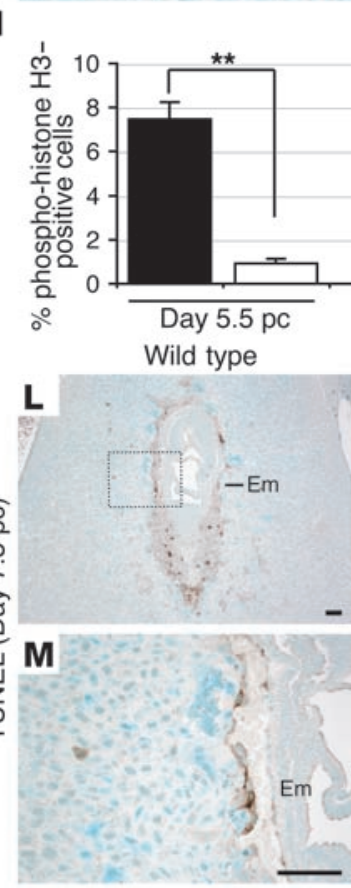

Sphk1-- Sphk2 ${ }^{+-}$

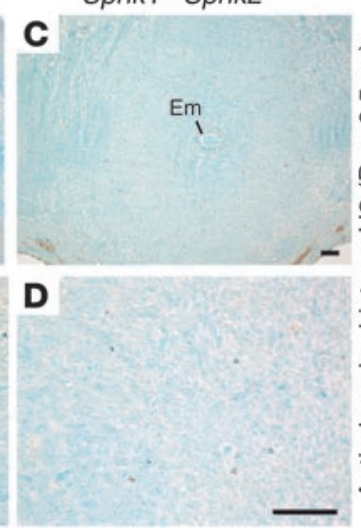

Wild type Sphk1 $1^{--}$Sphk2+/-
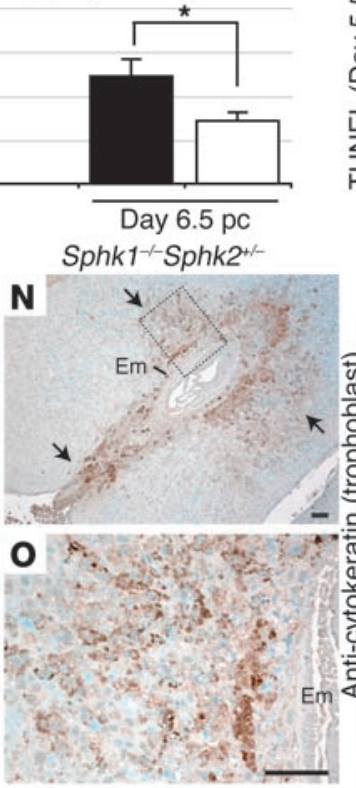
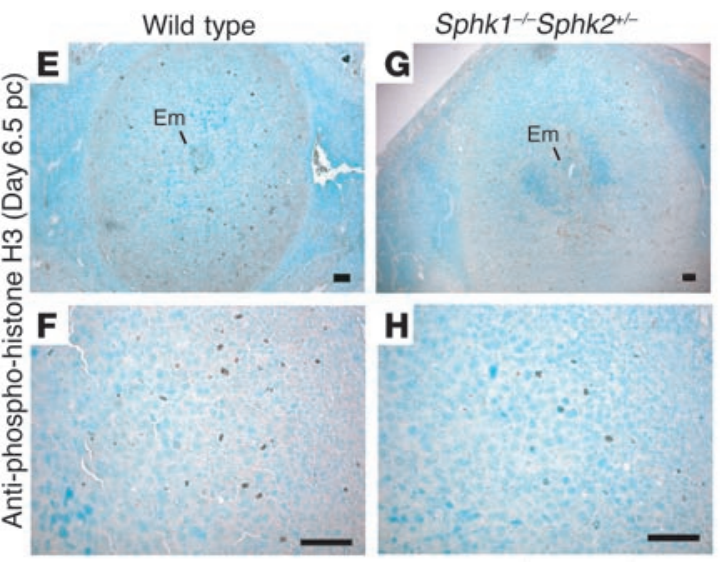

Wild type
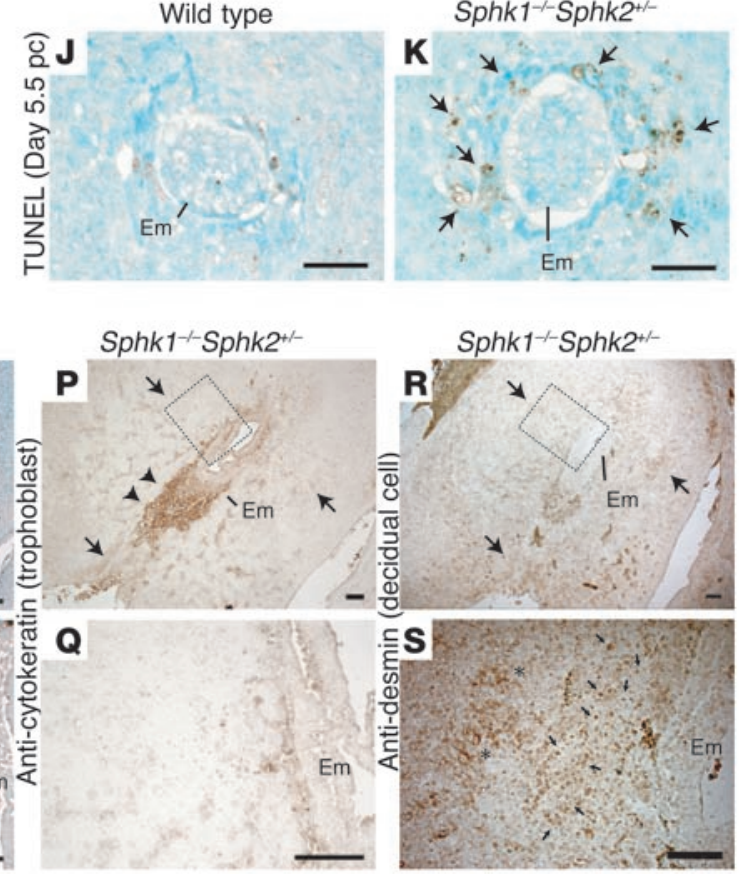

\section{Figure 4}

Decreased cell mitosis and increased cell death in Sphk1-/-Sphk2+/- decidua. (A-H) Immunostaining with anti-phospho-histone H3 on wild-type $(\mathbf{A}, \mathbf{B}, \mathbf{E}$, and F) and Sphk1-/-Sphk2+/- (C, D, G, and H) uteri on day 5.5 pc (A-D) and day 6.5 pc (E-H). (I) Percentage of phospho-histone H3-positive cells. $n=6$ matched pairs. ${ }^{*} P<0.05$; ${ }^{*} P<0.01$, paired Student's $t$ test. (J and K) TUNEL assay on wild-type (J) and Sphk1-/-

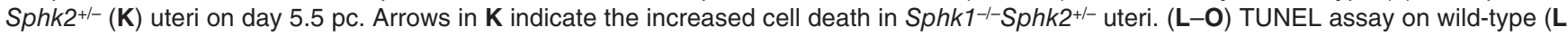
and $\mathbf{M}$ ) and Sphk1--Sphk2+- ( $\mathbf{N}$ and $\mathbf{O}$ ) uteri on day $7.5 \mathrm{pc}$. $\mathbf{M}$ and $\mathbf{O}$ represent high-power views of the boxed areas in $\mathbf{L}$ and $\mathbf{N}$, respectively. Arrows in $\mathbf{N}$ indicate the increased cell death in Sphk1--Sphk2+- decidua. (P and $\mathbf{Q})$ Immunostaining with anti-cytokeratin to label trophoblast cells on day 7.5 pc Sphk1--Sphk2+/- uteri. $\mathbf{Q}$ represents a high-power view of the boxed area in $\mathbf{P}$. Arrows in $\mathbf{P}$ correspond to those in $\mathbf{N}$. Note no positive staining in the area indicated by arrows. Arrowheads indicate positive trophoblast cells. (R and $\mathbf{S})$ Immunostaining with anti-desmin

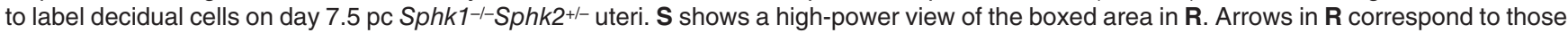
in N. Note shrunken decidual cells and normal decidual cells indicated by arrows and asterisks, respectively, in S. Scale bars: $100 \mu \mathrm{m}$ (A-H and $\mathbf{L}-\mathbf{S}) ; 50 \mu \mathrm{m}$ (J and $\mathbf{K})$. In all experiments, more than 3 animals were examined.

tion in the area surrounding the embryo, although the embryos were still viable (Figure 3, C-F). On day 7.5 pc, the hemorrhage and neutrophil infiltration in the decidual region were more noticeable (Figure 3, G-K), with necrotic embryos often observed (Figure 3K). The neutrophils were identified by immunostaining with an antineutrophil antibody (Figure 3L).

Sphk1 mRNA expression was examined in day 7.5 pc wild-type uteri by in situ hybridization. The Sphk1 mRNA was localized in the antimesometrial portion of the decidua, with more abundant expression in the secondary decidual zone (Figure 3, M and N), consistent with a role of $S p h k 1$ in decidualization.

Decidualization can be induced experimentally in pseudopregnant or steroid hormonally treated uteri by intraluminal oil infu-

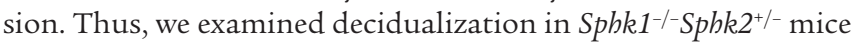
by intraluminal oil infusion on day $3.5 \mathrm{pc}$. Sphk $1^{-/-S p h k 2^{+/-} \text {mice }}$ showed a significantly lower decidual response than wild-type mice with respect to increased uterine weight $(4.3 \pm 3.1$ versus $18.4 \pm 4.2$ ) (Figure 3O). While 100\% (10/10) of the wild-type mice 

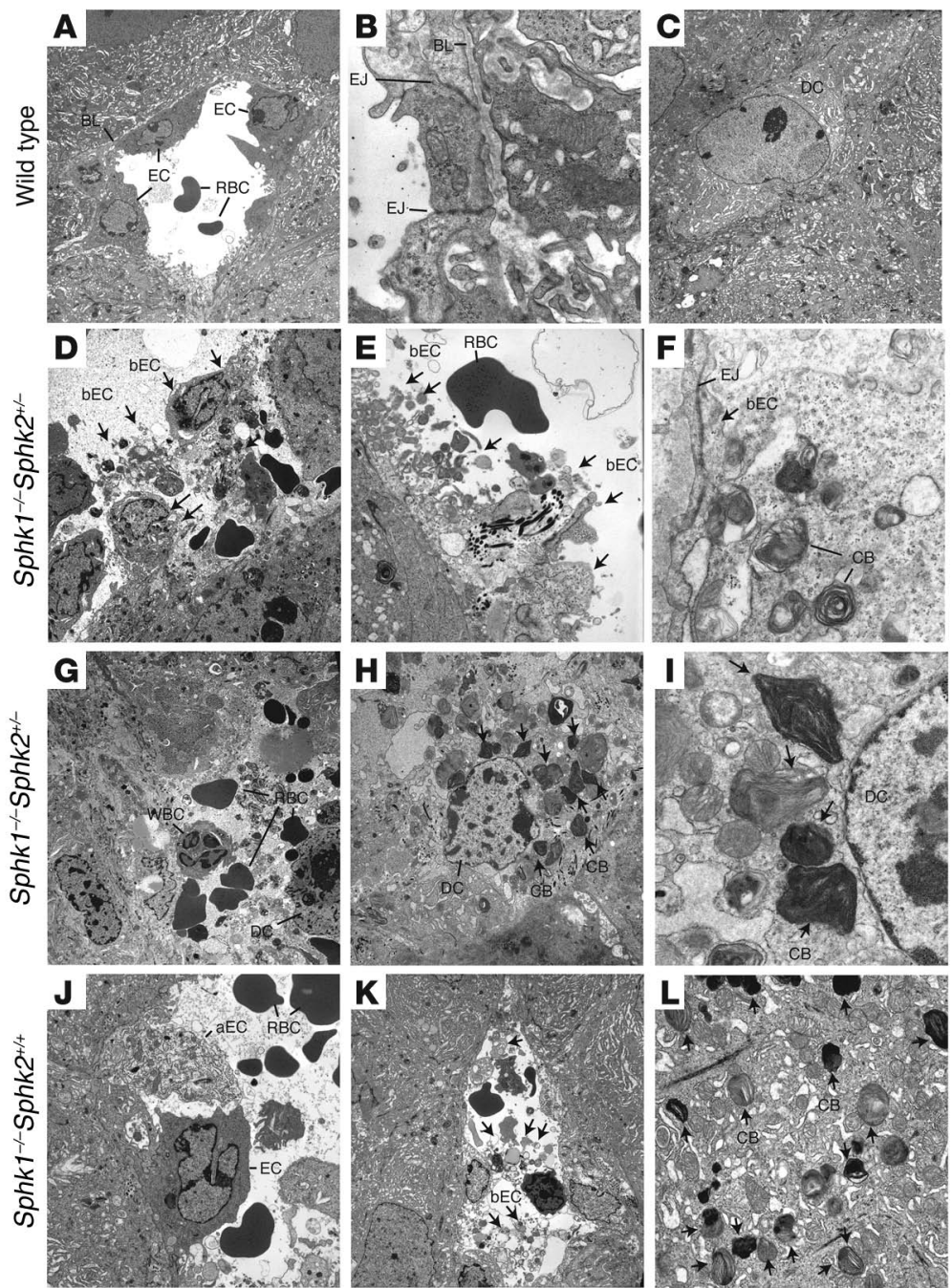
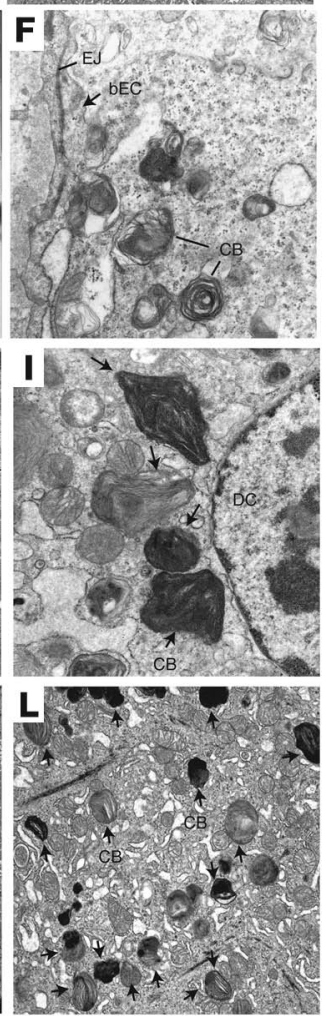

\section{Figure 5}

Transmission electron microscopic analysis of decidual cells and decidual blood vessels from wild-type (A-C),

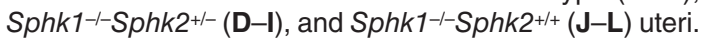
$\mathrm{aEC}$, aberrant endothelial cell with light and coarse cytoplasm; bEC, broken endothelial cell; BL, basal lamina; $\mathrm{CB}$; multilayered membranous cytoplasmic body; DC, decidual cell; EC, endothelial cell; EJ, endothelial cellcell junction; RBC, red blood cell; WBC, white blood cell. Arrows in $\mathbf{D}-\mathbf{F}$ and $\mathbf{K}$ indicate broken endothelial cells. Arrows in $\mathbf{H}, \mathbf{I}$, and $\mathbf{L}$ indicate multilayered membranous cytoplasmic bodies. Original magnification, $\times 2,000$ (A, C, D, G, H, and K); $\times 16,000$ (B, F, and I); $\times 6,300$ (E and L); and $\times 3,150(\mathbf{J})$.

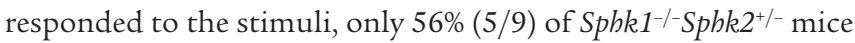
showed this response. Taken together, these results suggest a crucial role of Sphk in decidualization.

We assessed cell mitosis by anti-phosphohistone H3 immunostaining on day $5.5 \mathrm{pc}$ (Figure 4, A-D) and day $6.5 \mathrm{pc}$ (Figure $4, \mathrm{E}-\mathrm{H})$ uteri. The number of mitotic cells was significantly

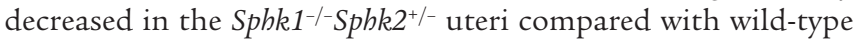
uteri at both stages (Figure 4I). We further studied cell survival of decidual cells in pregnant uteri. Apoptosis was assessed in wildtype and $S p h k 1^{-/} S p h k 2^{+/-}$uteri on day $5.5 \mathrm{pc}$ and day $7.5 \mathrm{pc}$ by the TUNEL assay. A mild increase in cell death was already observed in the decidual zone of day 5.5 pc Sphk1-/-Sphk2+/- uteri immediately surrounding the embryo, although they grossly looked

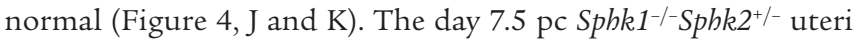
revealed remarkably increased apoptosis in the area surrounding the embryo, particularly in the primary decidual zone (Figure 4, $\mathrm{L}-\mathrm{O}$ ). To determine what cell types were undergoing increased cell death, immunostaining was performed using anti-cytokeratin and anti-desmin antibodies to label trophoblast cells and decidual cells, respectively (23-26). Desmin staining was observed throughout the decidual tissues, where shrunken, apparently dying cells were stained in the primary decidual zone (Figure 4, R and S). On the other hand, excessive trophoblast giant cell invasion was not observed in the decidual tissues, as denoted by the cytokeratin staining (Figure 4, P and Q). These results suggest that decidual cells are specifically undergoing increased cell death, which is not caused by increased trophoblast giant cell invasion.

Defects in decidual cells and decidual blood vessels in Sphk1-1-Sphk2+/uteri. Day 7.5 pc uteri of pregnant mice were further analyzed by electron microscopy. In Sphk1-/-Sphk2+/- uteri, all decidual blood vessels immediately surrounding embryos revealed severe endothelial cell breakage. Cellular organelles, such as the nucleus, mitochondria, ribosomes, and endoplastic reticulum as well as collagen fibers, were freely floating in the lumen of the blood vessels, and intact basal lamina and tight junctions were rarely detectable (Figure 5, D and E). Blood cell components from broken blood vessels were sometimes in contact with decidual cells directly (Figure 5G). Surprisingly, multilayered membranous cytoplasmic bodies, reminiscent of those found in sphingolipid storage diseases, were observed in the cytoplasm of decidual cells (Figure 5, H and I). The endothelial cells also included these cytoplasmic bodies, which were often found to be floating in the lumen of blood vessels (Figure 5F). Interestingly,

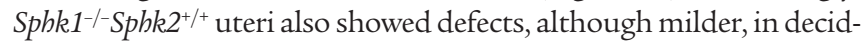
ual blood vessels and decidual cells, in spite of the fact that they

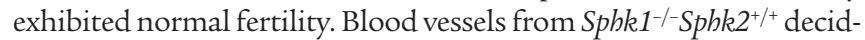
ua contained a mixture of nearly normal and aberrant endothelial 
A

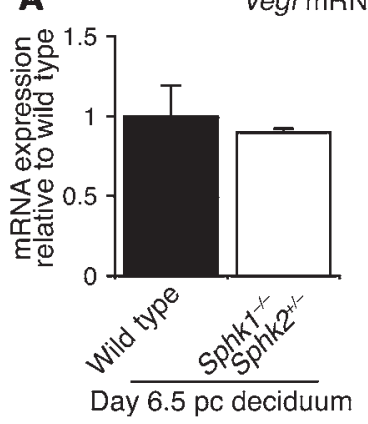

A expression
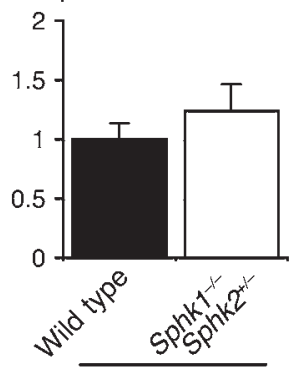

Day 7.5 pc deciduum

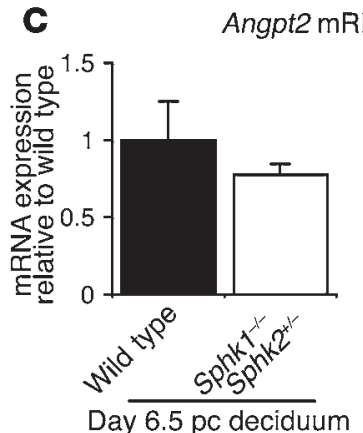

NA expression

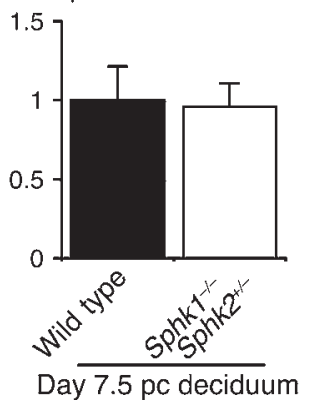

B

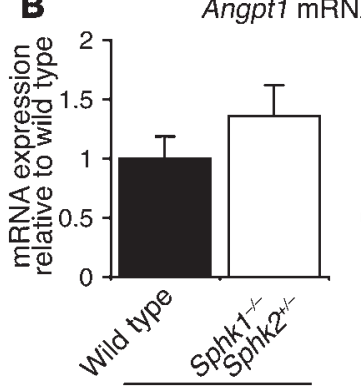

Day 6.5 pc deciduum
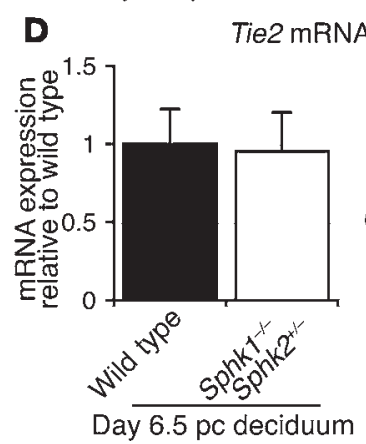

Figure 6

Vegf, Angpt1, Angpt2, and Tie2 mRNA expression. Vegf (A), Angpt1 (B), Angpt2 (C), and Tie2 (D) mRNA expression on day $6.5 \mathrm{pc}$ and day $7.5 \mathrm{pc}$ decidua from wildtype and Sphk1-/-Sphk2+/females as determined by real-time PCR. The expression levels are shown relative to those in wild-type decidua. Data represent mean values \pm SE. $n=3$. cells with light and coarse cytoplasm (Figure 5J). The endothelial

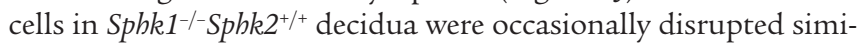

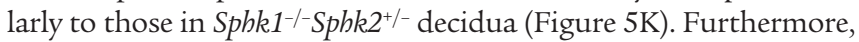

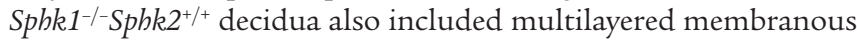
cytoplasmic bodies (Figure 5L) although they were less prominent

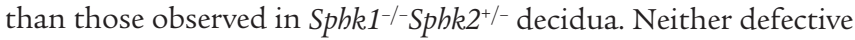
endothelial cells nor membranous cytoplasmic bodies were observed in wild-type decidua (Figure 5, A and C), and all wild-type blood vessels showed clear tight junctions and basal lamina (Figure 5B). The membranous cytoplasmic bodies were not found in any cells

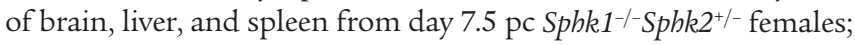
these are target organs of many sphingolipid storage diseases (Supplemental Figure 1; supplemental material available online with this article; doi:10.1172/JCI30674DS1). Blood vessels were also intact in these organs (Supplemental Figure 1). These data suggest that the cellular defects are specific to pregnant uteri.

We further examined the expression of factors implicated in angiogenesis, vascular endothelial growth factor (Vegf), angiopoietin 1 (Angpt1), angiopoietin 2 (Angpt2), and endothelial-specific receptor tyrosine kinase (Tie2) on day 6.5 pc and day 7.5 pc decid-

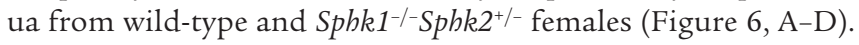
There was no difference in the expression levels of these factors in wild-type and Sphk1/-Sphk2+/- decidua either on day 6.5 pc or day $7.5 \mathrm{pc}$, indicating that these factors would not be the cause of disturbance in Sphk1-/-Sphk2+/- uteri.

The sphingolipid metabolic pathway is highly activated in the decidua during pregnancy. Total Sphk activity was measured in homogenates of nonpregnant whole uteri, day 7.5 pc decidua of pregnant mice, and day 7.5 pc interimplantation tissues (uterine tissues lacking implan-

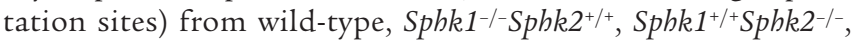
and $S p h k 1^{-/} S p h k 2^{+/-}$female mice, from which the embryos had been removed. Strikingly, Sphk activity was increased 9.8-fold in day 7.5 pc wild-type decidua of pregnant mice compared with that in day 7.5 pc wild-type interimplantation tissues in pregnant mice in the presence of Triton X-100 (Figure 7, A and B), which stimulates Sphk1 and inhibits Sphk2. Sphk activity was also increased in Sphk1 $1^{+/+} S p h k 2^{-/-}$

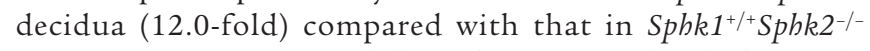
interimplantation tissues while Sphk activity was barely detectable

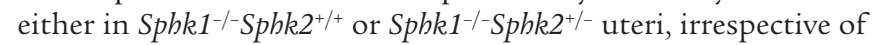
whether they were decidua or interimplantation tissues (Figure 7, $\mathrm{A}$ and B). Similar results were obtained in the absence of Triton $\mathrm{X}-100$, a condition in which both Sphk1 and Sphk2 are normally active, although Sphk activity was detectable to some extent in

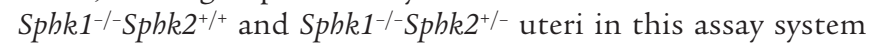
(Figure 7, C and D). Nonpregnant whole uteri showed a trend of Sphk activity similar to that of interimplantation tissues in all genotypes of mice (Figure 7, A-D). These results suggest that Sphk activity was dramatically increased during pregnancy in wild-type and Sphk1+/+Sphk2-/- decidua whereas it was not inducible during

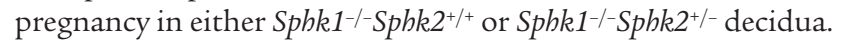

To investigate sphingolipid metabolism in normal pregnancy, we examined the mRNA levels for major genes involved in the sphingolipid metabolic pathway (Figure 8) in nonpregnant whole uteri, day 7.5 pc decidua of pregnant mice, and day 7.5 pc interimplantation

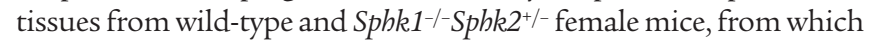
the embryos had been removed. In line with Sphk activity, the Sphk1 mRNA levels were 9.3-fold higher in day 7.5 pc wild-type decidua of pregnant mice than in day $7.5 \mathrm{pc}$ wild-type interimplantation tissues while the Sphk2 mRNA levels were not significantly altered (Figure 9A). The expression of other key genes (Figure 8) was also upregulated in pregnant wild-type decidua compared with pregnant wild-type interimplantation tissues: serine palmitoyl transferase 1 and 2 (Sptlc1, 8.7-fold; Sptlc2, 6.3-fold), S1P lyase (Sgpl1; 11.3fold), S1P phosphatase 1 (Sgpp1; 7.7-fold), and sphingomyelinase 1 (Smpd1; 5.3-fold) (Figure 9A). The mRNA levels of the genes involved in the sphingolipid pathway, other than Sphk1 and Sphk2, were also

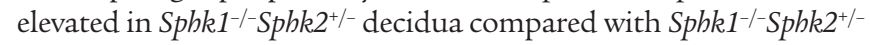
interimplantation tissues and did not significantly differ from those in wild-type decidua (Figure 9A). Interestingly, the Lass genes, which encode dihydroceramide synthases (27), were not highly elevated in 
A

+ Triton X-100

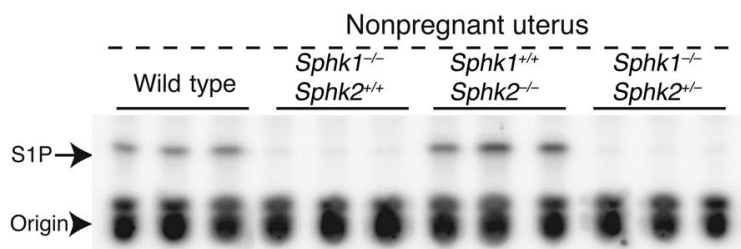

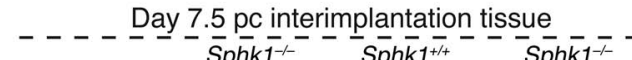
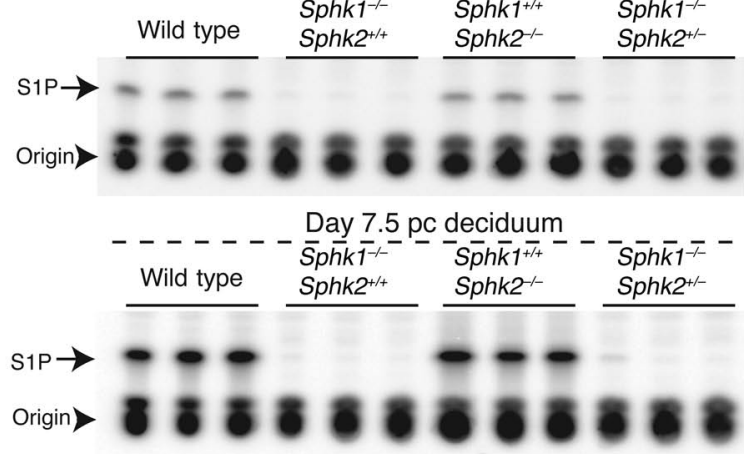

B Nonpregnant uterus

$\square$ Day 7.5 pc interimplantation tissue Day 7.5 pc deciduum

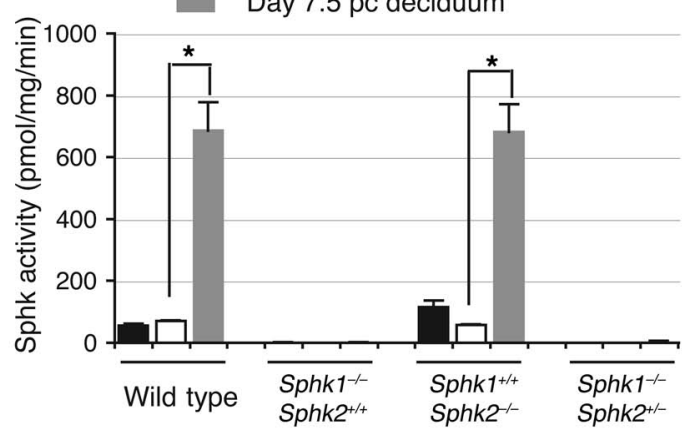

C

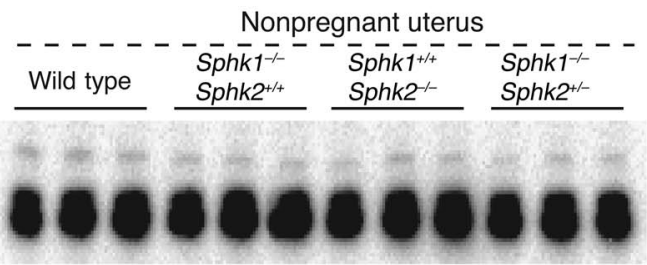

Day $7.5 \mathrm{pc}$ interimplantation tissue

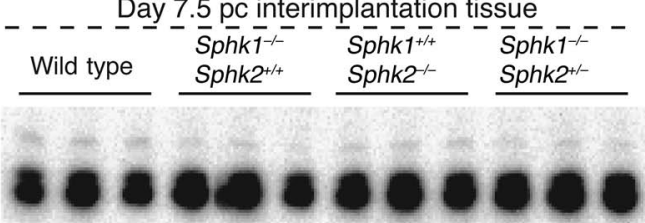

Day 7.5 pc deciduum

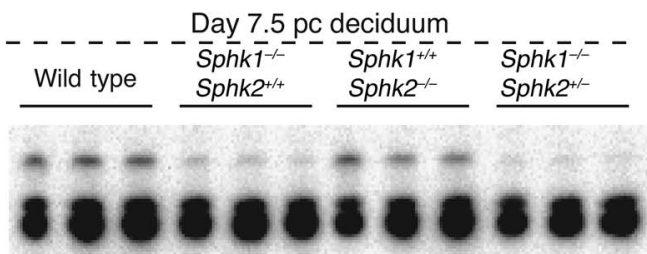

D Nonpregnant uterus

Day 7.5 pc interimplantation tissue

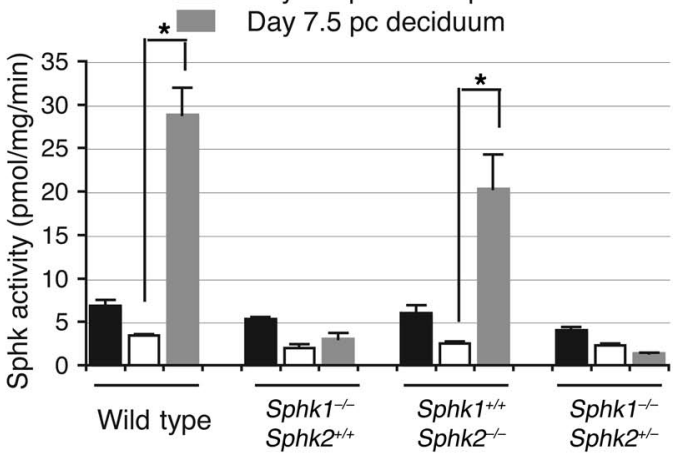

Figure 7

Measurement of Sphk activity. Sphk enzymatic activity was determined in nonpregnant whole uteri, day 7.5 pc decidua, and day 7.5 pc interim-

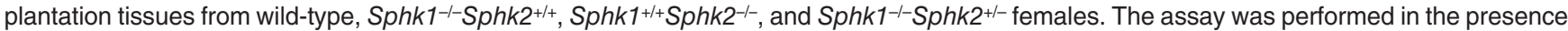
of Triton X-100 (A and B) or of BSA complexes without Triton X-100 (C and D). (A and C) TLC analysis of [32P] S1P formed by Sphk in uterine samples. The bands for S1P and origin are shown. (B and D) Sphk activity. The data represent mean values \pm SE and were compared between day 7.5 pc decidua and day 7.5 pc interimplantation tissues. $n=3 .{ }^{*} P<0.05$, paired Student's $t$ test.

the wild-type decidua during pregnancy compared with interimplantation tissues although the expression levels of the Lass 5 and Lass 6 were slightly increased (1.4-fold and 2.8-fold, respectively) (Figure 9B). The expression profile of nonpregnant whole uteri was similar to that of interimplantation tissues in both wild-type and Sphk1-/-Sphk2+/- mice (Figure 9, A and B). Furthermore, day 7.5 pc

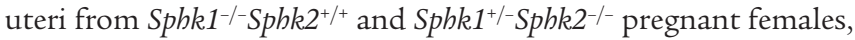
both of which are fertile, exhibited a similar expression profile to the wild-type uteri, except for the lack of Sphk1 and Sphk2 expression, respectively (Supplemental Figure 2, A and B). Taken together, these results suggest that the de novo synthesis pathway for sphingolipids, in particular the portion leading to the production and degradation of S1P, is highly activated at the transcription level in the decidua during pregnancy and that the reproductive phenotype observed in $S p h k 1^{-/} S p h k 2^{+/-}$females can be mainly attributed to the Sphk1 expression, but with a dosage effect of Sphk2.
Sphingoid bases abnormally accumulate in pregnant Sphk1-/-Sphk2+/decidua. Sphingolipid levels were measured by mass spectrometry in homogenates of nonpregnant whole uteri (wild-type and Sphk1/-

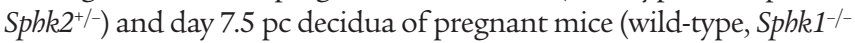

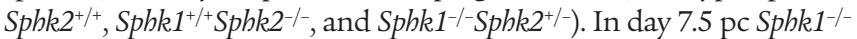

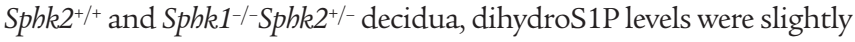
higher than in day 7.5 pc wild-type decidua (1.6-fold and 1.9-fold, respectively) (Figure 10A). S1P levels were not significantly altered in all types of pregnant decidua examined (Figure 10B). In sharp contrast, both dihydrosphingosine (sphinganine) and sphingosine levels were substantially increased (70.5-fold and 18.0-fold, respectively) in day 7.5 pc Sphk1 1/-Sphk2+/- decidua as compared with those in day $7.5 \mathrm{pc}$ wild-type decidua (Figure 10, C and D). Higher levels of sphingoid bases were also observed in $S p h k 1^{-/-S p h k 2^{+/+}}$decidua, although less

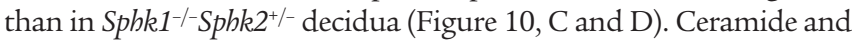
sphingomyelin levels were not significantly different in all types of day 


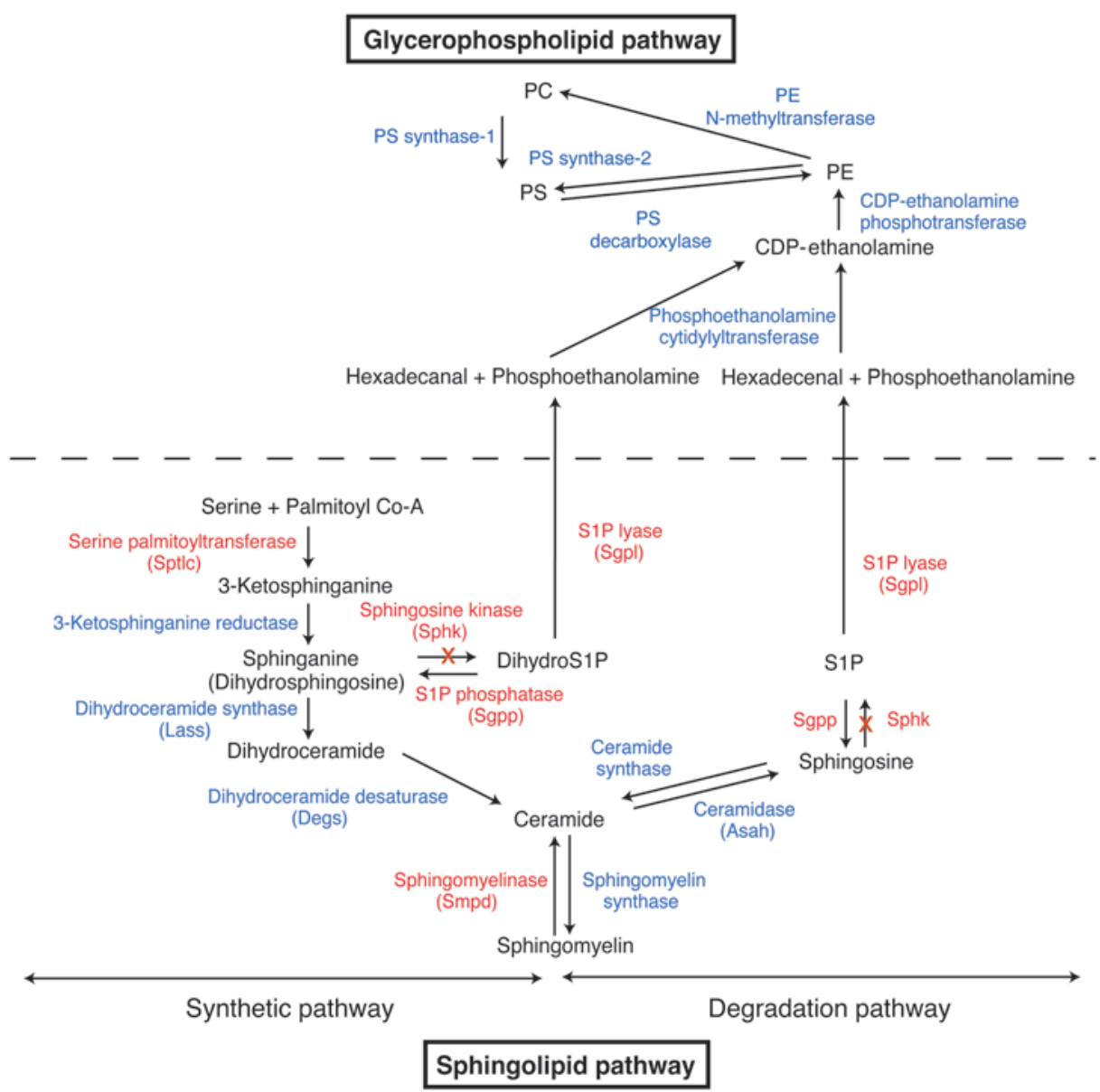

Figure 8

Sphingolipid and related glycerophospholipid biosynthetic pathways. Enzymes, expression levels of which are increased during pregnancy, are indicated in red color. $\mathrm{X}$, genetic disruption of Sphk genes.
7.5 pc decidua examined (Figure 10, E and F). In summary, pregnant

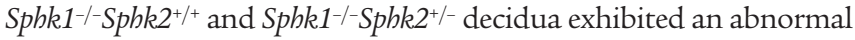
accumulation of dihydrosphingosine and sphingosine, which was

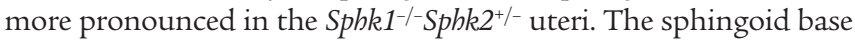
accumulation can be attributed to the defective Sphk activity in the pregnant uteri. From these results, it is possible that the membranous cytoplasmic bodies observed in the electron microscopic analysis resulted from sphingosine and dihydrosphingosine accumulation. These results obtained by mass spectrometry were confirmed by alternative methods described previously (data not shown) $(19,28)$.

Phosphatidylethanolamine levels are reduced in Sphk1-/-Sphk2+/- decidua. Next, we measured levels of the glycerophospholipids cardiolipin (CL), phosphatidylcholine (PC), phosphatidylethanolamine (PE), and phosphatidylserine (PS) in nonpregnant whole uteri (wild-type and Sphk1 $1^{-/} S p h k 2^{+-}$) and day 7.5 pc decidua of pregnant mice (wild-type and $\left.S p h k 1^{-/} S p h k 2^{+-}\right)$, from which the embryos had been removed. In

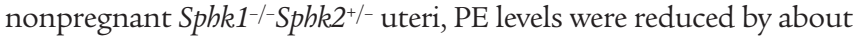
$37 \%$ compared with nonpregnant wild-type uteri (Figure 11C). A simi-

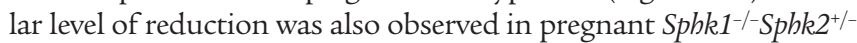
decidua compared with pregnant wild-type decidua (Figure 11C). S1P and dihydroS1P were degraded by S1P lyase to produce fatty aldehyde and phosphoethanolamine, which were then reused for biosynthesis

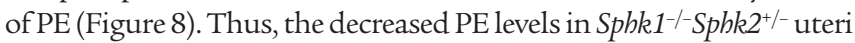
would be reasonable to expect if products derived from the sphingolipid metabolic pathway were being utilized for PE biosynthesis. The levels of CL, PC, and PS were not significantly different in the 4 types of uteri examined (Figure 11, A, B, and D). There were no significant differences in the levels of CL, PC, PE, and PS between day 6.5 pc wildtype and Sphk1 $1^{-/}$Sphk2 $2^{+-}$decidua (Supplemental Figure 3, A-D). These results indicate that the diminution of PE levels observed in day $7.5 \mathrm{pc}$ Sphk1/-Sphk2+- decidua is present when defective decidualization is apparent. Thus, the PE deficiency may accelerate the defect leading to the pregnancy loss, rather than causing the primary defect.

The PG pathway is not involved in the pathogenesis of Sphk1-/-Sphk2+/uteri. The PG pathway plays crucial roles in female reproduction. PG-endoperoxidase synthase-2 (Ptgs2), also known as COX2, converts arachidonic acid to $\mathrm{PG} \mathrm{H}_{2}\left(\mathrm{PGH}_{2}\right)$ and is a key enzyme in the biosynthesis of PGs. The $\mathrm{PGH}_{2}$ is then converted to various PGs by specific enzymes. Deficiency of Ptgs 2 causes multiple female reproductive failures, including defective attachment reaction and defective decidualization in mice (29). Thus, we examined the Ptgs2 expression and prostaglandin $\mathrm{E}_{2}\left(\mathrm{PGE}_{2}\right)$ levels in day $6.5 \mathrm{pc}$ and day

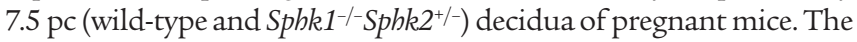
Ptgs 2 expression in Sphk1-/-Sphk2+/- decidua did not significantly differ from that of wild-type decidua, either on day 6.5 pc or day 7.5 pc (Figure 12A). The parallel result was obtained with $\mathrm{PGE}_{2}$ levels, where there was no difference between wild-type and Sphk1-/-Sphk2+decidua (Figure 12B). These results suggest that PG biosynthesis is unlikely to be involved in the pathogenesis of Sphk1-/-Sphk2+/- uteri.

\section{Discussion}

Sphingolipids are implicated in diverse biological functions. In this study, we have discovered that the sphingolipid metabolic pathway is highly activated in the decidua during normal pregnancy. The 
A

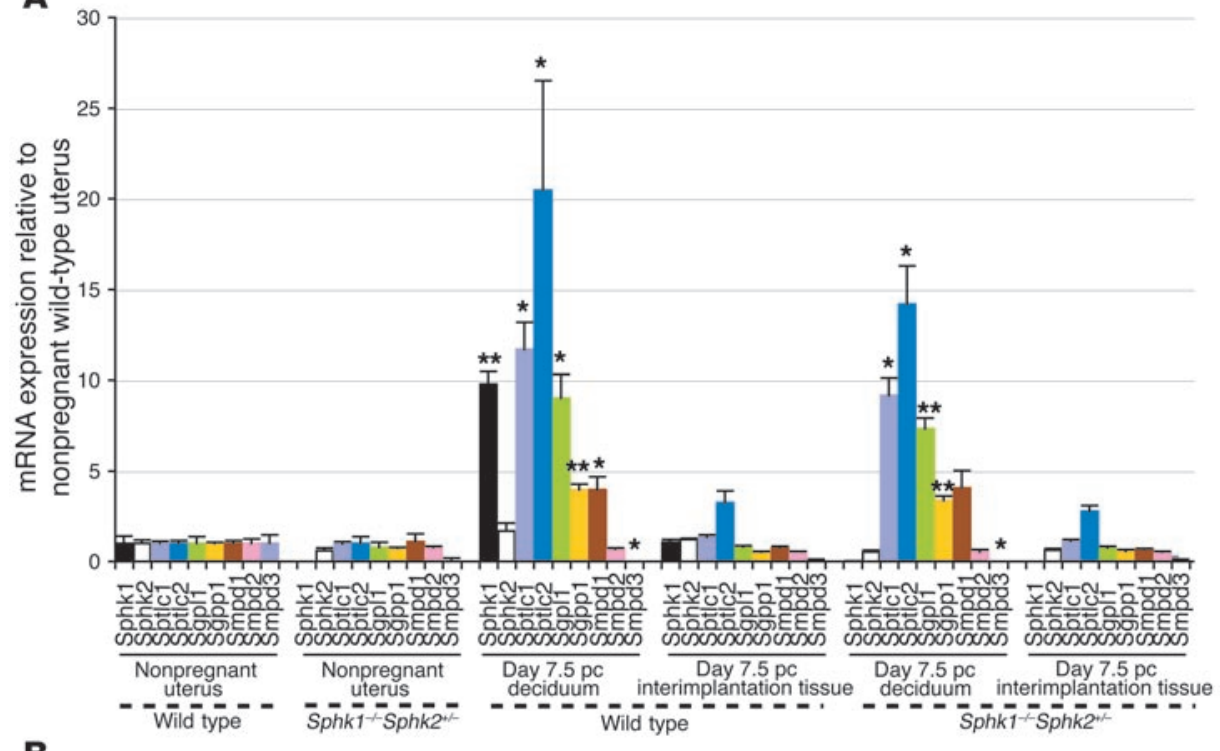

B

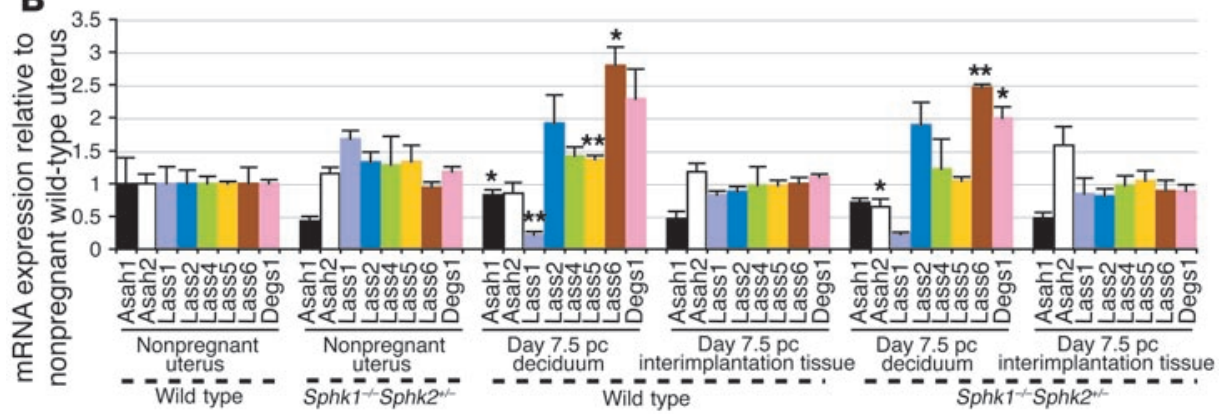

Figure 9

The expression levels of enzymes involved in sphingolipid metabolism during pregnancy. Relative mRNA expression of Sphk1, Sphk2, Sptlc1, Sptlc2, Sgpl1, Sgpp1, Smpd1, Smpd2, Smpd3, Asah1, Asah2, Lass1, Lass2, Lass4, Lass5, Lass6, and Degs1 in nonpregnant whole uteri, day $7.5 \mathrm{pc}$ decidua of pregnant mice, and day 7.5 pc interimplantation tissues from wildtype and Sphk1---Sphk2+-- females as determined by real-time PCR. Expression levels are shown relative to those in nonpregnant wild-type uteri. Data represent mean values \pm SE and were compared between day $7.5 \mathrm{pc}$ decidua and day $7.5 \mathrm{pc}$ interimplantation tissues. $n=3 .{ }^{\star} P<0.05 ;{ }^{\star \star} P<0.01$, paired Student's $t$ test. maternal disturbance in the activated pathway by disruption of Sphk genes causes sphingoid base accumulation and a reduction of PE levels, followed by severe defects in decidual cells and decidual blood vessels, leading to maternally derived early pregnancy loss.

The importance of sphingolipids in other aspects of the female reproductive system has been reported. Disruption of the acid sphingomyelinase gene, which mediates hydrolysis of sphingomyelin to yield ceramide, suppresses oocyte apoptosis (30). Furthermore, oocyte apoptosis induced by anticancer therapy in wild-type female mice was prevented by pretreatment of ovaries with S1P, without any genomic damage to the offspring $(30,31)$. These findings have established ceramide as being proapoptotic, and S1P as being antiapoptotic in oocytes. We observed severely compromised fertility in

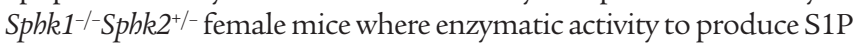
was markedly reduced. However, those female mice did not show any obvious abnormalities in the ovaries or during the early implantation process for fertilized embryos. Instead, uterine decidualization was severely impaired in these females. Electron microscopic analysis of the decidua revealed that multilayered membranous cytoplasmic bodies accumulated within the cytoplasm of decidual cells and vascular endothelial cells in the decidua, leading to breakage of endothelial cells with blebbing of cellular components into the vessel lumen. Based on the sphingolipid analysis showing excessive accumulation of sphingoid bases, these cytoplasmic bodies may contain sphingosine and dihydrosphingosine. The defects observed in vascular endothelial cells would likely cause hemorrhage followed by infiltration of inflammatory cells and increased cell death in the decidua.
These consequences were in fact observed by histological examination. Under these circumstances, the defective decidual blood flow would be unable to nourish embryos sufficiently and would block trophoblast cells from invading the maternal vasculature, which is imperative for the establishment of placenta. In rodents, decidual cells and trophoblast cells produce the prolactin family of hormones, which target the mammary gland and corpus luteum during pregnancy $(32,33)$. These hormones act to maintain the corpus luteum of pregnancy and to stimulate luteal $\mathrm{P}_{4}$ production. The orchestrated expression of these hormones by decidual cells and trophoblast cells ensures its continuous presence from the implantation until parturition, suggesting its physiological importance during pregnancy. In our mutant mice, although the decidualization was severely defective, the $\mathrm{P}_{4}$ secretion was retained during pregnancy.

Sphingoid bases can be proapoptotic as is the case for ceramide, in contrast with the antiapoptotic effect of S1P. Fumonisins, which are mycotoxins that inhibit dihydroceramide synthase, cause accumulation of sphinganine and result in cytotoxicity (34). Treatment of prostate cancer cells with exogenous sphingosine or the Sphk inhibitor $N, N$-dimethylsphingosine induces apoptosis (35). Furthermore, doxorubicin treatment of MCF7 breast cancer cells increases sphingosine levels followed by mitochondrial cytochrome $c$ release, caspase activation, and apoptosis. These proapoptotic effects of doxorubicin can be mimicked by exogenous sphingosine (36). Thus, we believe that sphingoid base accumulation would

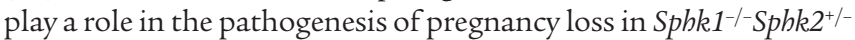
female mice. Recently, it has been reported that extracellular 
A

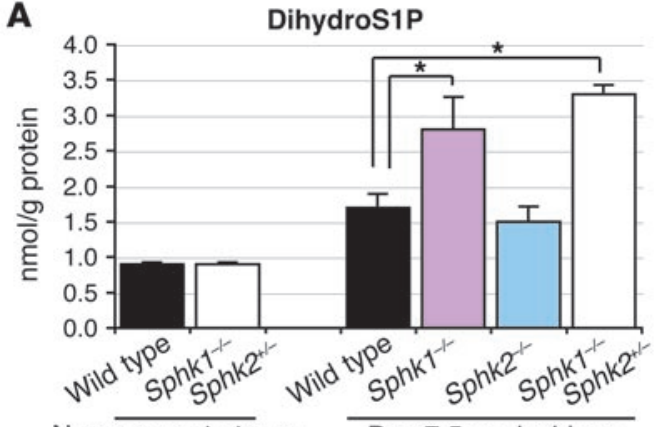

Nonpregnant uterus

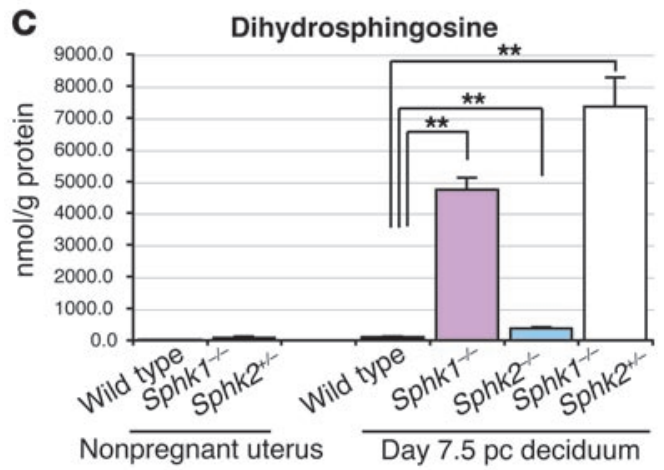

E

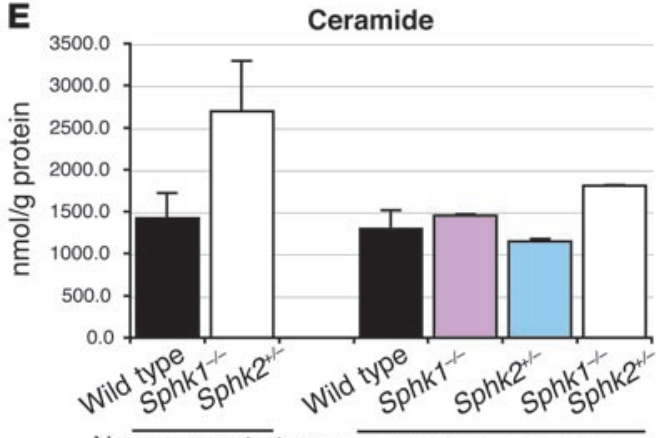

B
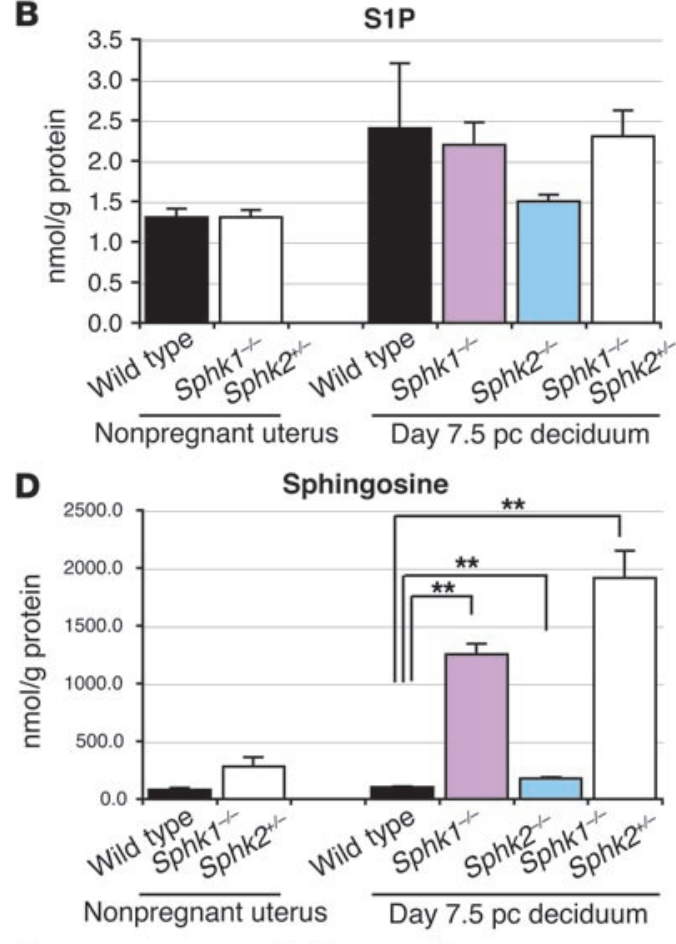

F

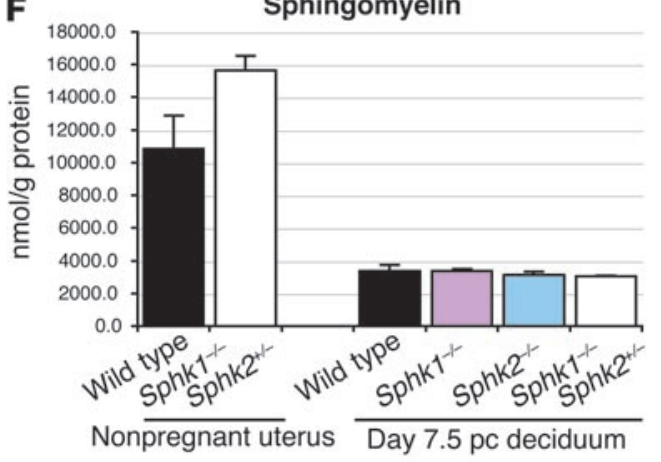

Figure 10

Measurement of sphingolipid levels. DihydroS1P (A), S1P (B), dihydrosphingosine (C), sphingosine (D), ceramide (E), and sphingomyelin (F) levels were determined in nonpregnant whole uteri (wild type and Sphk1-/-Sphk2+/-) and day $7.5 \mathrm{pc}$ decidua (wild type, Sphk1-/-Sphk2+/+, Sphk1+l+Sphk2-l-, and

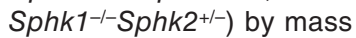
spectrometry. Data represent mean values \pm SE. $n=3$. ${ }^{\star} P<0.05$; ${ }^{\star \star} P<0.01$, paired Student's $t$ test.
S1P could be converted to intracellular S1P by lipid phosphate phosphatase-1 (LPP-1) and Sphk1 in human lung endothelial cells (37). Thus, it also may be possible that intracellular S1P levels are reduced in $S p h k 1^{-/} S p h k 2^{+/-}$female mice, contributing to the increased cell death in the decidua, although overall S1P levels are not significantly different from those in wild-type mice. Interest-

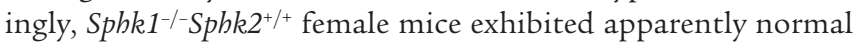
fertility in spite of the substantial sphingoid base accumulation

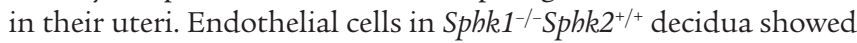

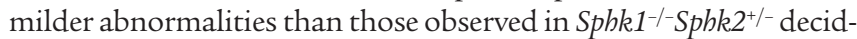
ua, indicating that the appearance of phenotype may be dependent on the severity of endothelial cell defects. The results suggest that a

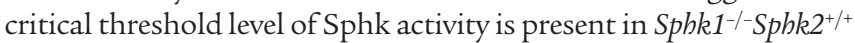
mice that enables pregnancy to proceed and that the Sphk activity

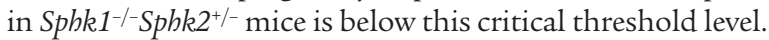

It is noteworthy that PG biosynthesis is unlikely to be implicated in the pathogenesis of pregnancy loss associated with Sphk1-/$S p h k 2^{+/-}$uteri. Lysophosphatidic acid (LPA) is a bioactive phospholipid related to S1P. Recently, the G protein-coupled receptor $\mathrm{LPA}_{3}$ has been reported to be crucial for embryo implantation and spacing (38). The $l p a_{3}$-deficient females showed deferred implantation, which led to inappropriate embryo spacing and reduced litter size. In $\mathrm{Ppa}_{3}{ }^{-1-}$ females, levels of Ptgs 2 expression and PGs were reduced, but treatment of mice with PGs resumed on-time implantation, suggesting linkage between LPA signaling and PG biosynthesis (38). Furthermore, the expression of $S 1 P$ receptors $\left(S 1 P_{1}\right.$ and $\left.S 1 P_{2}\right)$ in the decidual microvasculature was found to overlap with Ptgs2 expression, and treatment of predecidualized stromal cells with S1P induced Ptgs2 expression, indicating a link between sphingolipid- and PG-signaling pathways (39). Based on the present data, which show normal Ptgs2 expression and $\mathrm{PGE}_{2}$ levels in pregnant Sphk1-/-Sphk2+/- uteri, we favor a pathogenic mechanism for inducing pregnancy loss not related to the PG pathway.

What is the importance of the activated sphingolipid metabolic pathway in normal pregnancy? S1P receptor signaling may provide important signals for decidualization and angiogenesis (39). As described above, it has been suggested that S1P receptor signaling is linked to the PG signaling pathway. Thus, generation of the receptor ligands S1P and dihydroS1P may be a consequence of activated sphingolipid metabolism during pregnancy. Although only slightly increased during pregnancy, the levels of these signaling lipids may be under tight control. Another function of the metabolic path- 

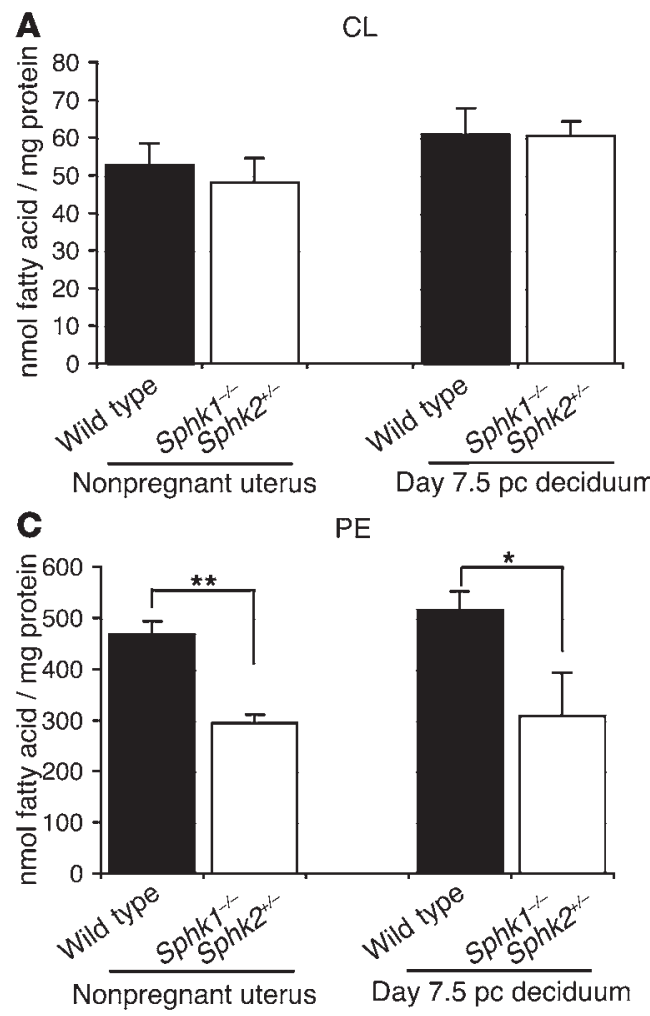

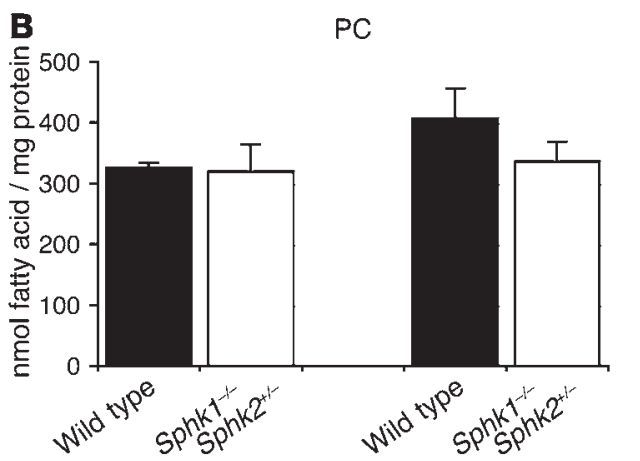

\section{Figure 11}

Measurement of glycerophospholipid levels. CL (A), PC (B), PE (C), and PS (D) levels were determined in nonpregnant whole uteri and day $7.5 \mathrm{pc}$ decidua from wild-type and Sphk $1^{-/-}$ Sphk2+/- pregnant female mice. Data represent mean values \pm SE. $n=3 .{ }^{* \star} P<0.05,{ }^{*} P=0.05$, paired Student's $t$ test.

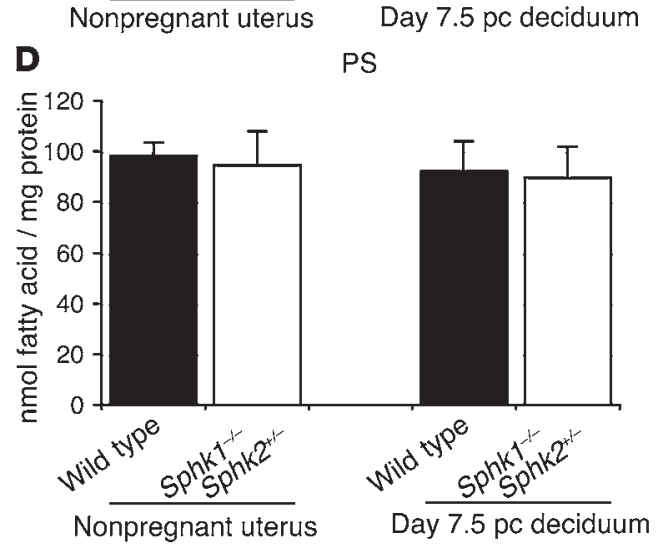

way might be revealed by the finding that PE levels were reduced

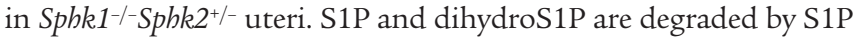
lyase to produce fatty aldehyde and phosphoethanolamine, which then can be reused for biosynthesis of PE. Thus, the decreased levels of PE in Sphk1-/-Sphk2+-- uteri could be the result of the block of substrate flux through the sphingolipid metabolic pathway. $\mathrm{PE}$ is a major component of both the outer and inner leaflets of cell plasma membranes and makes a complex with several plasma proteins, such as high-molecular weight kininogen, low-molecular weight kininogen, and proteins in complex with high-molecular weight kininogen, factor XI, or prekallikrein $(40,41)$. Kininogen and its metabolites are abundantly expressed in the female reproductive system (42-45). Furthermore, in antiphospholipid syndrome, which is clinically characterized by systemic thrombosis and recurrent pregnancy loss, anti-PE antibody recognizes the PE-binding proteins $(40,41)$. Kininogen and prekallikrein/kallikrein were found to be expressed in the endothelial cells of the placental villous capillaries, suggesting their importance in the vasculature of fetomaternal interface (45). Biologically active kinins generally exert their functions in vasodilation and vasoconstriction, and regulate blood flow. Furthermore, it has recently been reported that ethanolamine kinase 2-deficient female mice, in which PE levels are reduced, exhibited fetal death at the late stage of pregnancy due to placental thrombosis (46). Thus, $\mathrm{PE}$ biosynthesis, which is of particular importance during pregnancy, may require an activated sphingolipid metabolic pathway to provide precursors. We speculate that a reduced level of the PE-kininogen complex could also contribute to the loss of vascular stability in the fetomaternal interface and contribute to pregnancy loss.

Our findings also have potential clinical implications. Recently, it has been reported that S1P inhibits cytotrophoblast differentiation and could be involved in the pathogenesis of preeclampsia in humans (47). Furthermore, S1P has been shown to induce a dose-dependent vasoconstriction in human uterine arteries during pregnancy (48). Given these results and the results in our study, disturbance in sphingolipid metabolism may be relevant to human reproductive failure.

\section{Methods}

Mice. Disruption of the Sphk1 and Sphk2 genes was performed as described $(12,21)$. Mice were maintained on a mixed genetic background, C57BL/6× 129Sv. In all experiments, females were mated with wild-type males, and the day of the vaginal plug was considered day $0.5 \mathrm{pc}$. Animal care and use was approved by the NIH Animal Research Advisory committee, and all the mice used were housed at the Animal Care Facility at the NIH.

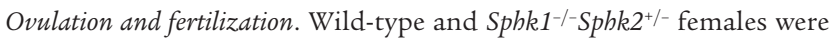
mated with fertile wild-type males. The females were sacrificed on day $1.5 \mathrm{pc}$ and oviducts were flushed with Brinster's BMOC-3 Medium (Invitrogen) to recover eggs and embryos. Their morphology was examined under a dissection microscope.

Blue dye reaction. For the blue dye reaction, $0.1 \mathrm{ml}$ Evans blue dye (1\% in PBS) was injected into the tail vein of mice on day $5.5 \mathrm{pc}$. Mice were sacrificed 5 minutes later and uteri inspected for the presence of blue bands.

Embryo transfer. Blastocysts were flushed from day 3.5 pc uteri of 3-weekold wild-type donors that had been superovulated. Females were mated with vasectomized males to induce pseudopregnancy. Seven to nine blastocysts were transferred to each uterine horn of wild-type or $S p h k 1^{-/-} S p h k 2^{+/-}$pseudopregnant recipients on day $2.5 \mathrm{pc}$. Recipients were sacrificed on day $8.5 \mathrm{pc}$.

Decidualization. To induce artificial decidualization, 1 uterine horn of wild-type or Sphk1-/-Sphk2+/- mice was infused intraluminally with $15 \mu \mathrm{l}$ of sesame oil (Sigma-Aldrich) on day 3.5 pc of pseudopregnancy. The noninfused uterine horn served as a control. The mice were sacrificed 4 days after the deciduogenic stimulus. Uterine weights of the infused and noninfused horn were recorded, and the fold increases in uterine weights were used as an index of decidualization. 
A

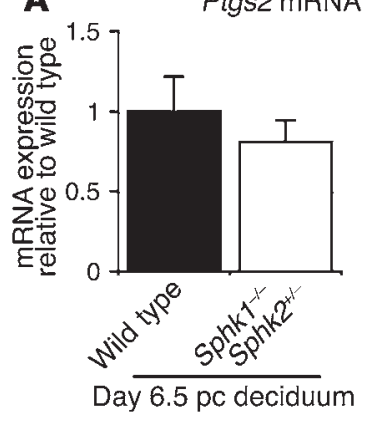

B

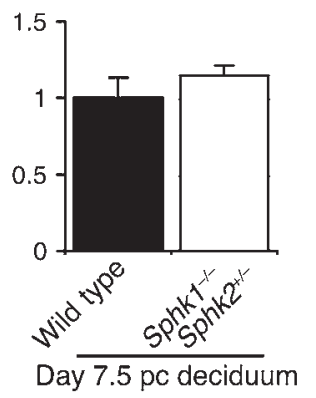

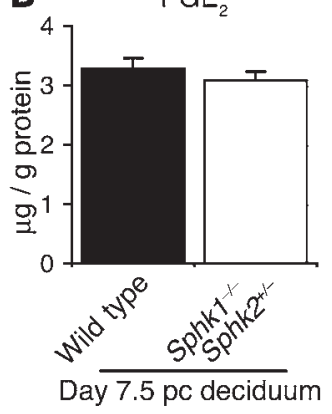

Figure 12

Ptgs2 mRNA expression and $\mathrm{PGE}_{2}$ levels. (A) Ptgs2 mRNA expression on day $6.5 \mathrm{pc}$ and day 7.5 pc decidua from wild-type and Sphk1-/-Sphk2+/females as determined by real-time PCR. The expression levels are shown relative to those in wild-type decidua. (B) $\mathrm{PGE}_{2}$ levels in day $7.5 \mathrm{pc}$ decidua from wild-type and Sphk1-/-Sphk2+/- pregnant female mice. The data represent mean values \pm SE. $n=3$.
Hormone injections. To determine whether Sphk responds to estrogen $\left(\mathrm{E}_{2}\right)$ and/or $\mathrm{P}_{4}$, 8-week-old mice were ovariectomized and rested for 2 weeks before they received any injections. Mice were injected with $\mathrm{E}_{2},(100 \mathrm{ng} / \mathrm{mouse})$, $\mathrm{P}_{4}\left(2 \mathrm{mg} /\right.$ mouse), or $\mathrm{E}_{2}$ plus $\mathrm{P}_{4}$. The control mice received sesame oil $(0.1 \mathrm{ml} /$ mouse). They were sacrificed 6 or 12 hours after the last injection, and uteri were collected for the measurement of Sphk activity. The hormones were dissolved in sesame oil and injected $(0.1 \mathrm{ml} /$ mouse) subcutaneously. Another group of mice received a regimen to mimic the estrous cycle and early pregnancy: $\mathrm{E}_{2}(100 \mathrm{ng})$ for 3 days (days $\left.1-3\right)$, no treatment on days 4 and 5 , and $\mathrm{P}_{4}(1 \mathrm{mg})$ plus $\mathrm{E}_{2}(10 \mathrm{ng})$ for 5 days (days 6-10). On day 10 , uteri were collected and subjected to Sphk assay.

Circulating serum $P_{4}$ levels. Serum was harvested from whole blood collect-

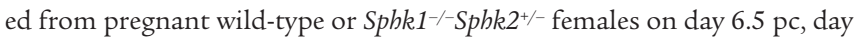
$7.5 \mathrm{pc}$, day $8.5 \mathrm{pc}$, or day $11.5 \mathrm{pc}$. The $\mathrm{P}_{4}$ levels were determined using the Enzyme Immunoassay for Progesterone (Oxford Biochemical Research) according to the manufacturer's instructions.

Quantitative real-time PCR. Total RNA from nonpregnant whole uteri, decidua of day $6.5 \mathrm{pc}$ and $7.5 \mathrm{pc}$ uteri, and interimplantation tissues of day $6.5 \mathrm{pc}$ and $7.5 \mathrm{pc}$ uteri, from which the embryos had been removed, was isolated using TRIzOL (Invitrogen). Total RNA $(1 \mu \mathrm{g})$ was reverse-transcribed using the SuperScript First-Strand Synthesis System for RT-PCR (Invitrogen) following the manufacturer's instructions. Mouse Sphk1, Sphk2, Sgpp1, Sgpl1, Sptlc1, Sptlc2, Smpd1, Smpd2, Smpd3, Asah1, Asah2, Lass1, Lass2, Lass4, Lass5, Lass6, Degs1, Ptgs2, Vegf, Angpt1, Angpt2, and Tie2 mRNA was quantified using Assays-on-Demand (Applied Biosystems) with an ABI Prism 7700 Sequence Detection System (Applied Biosystems). $\beta$-actin was used as an internal standard.

Measurement of Sphk activity. Sphk activity was measured essentially as described previously (49). Homogenates (20 $\mu$ g protein) from nonpregnant whole uteri and day $7.5 \mathrm{pc}$ decidua or interimplantation tissues, from which the embryos had been removed, in Sphk buffer $(50 \mathrm{mM}$ Tris, $\mathrm{pH} 7.5,10 \%$ glycerol, $1 \mathrm{mM} \beta$-mercaptoethanol, $1 \mathrm{mM}$ EDTA, $1 \mathrm{mM}$ sodium orthovanadate, $40 \mathrm{mM} \beta$-glycerophosphate, $15 \mathrm{mM} \mathrm{NaF}, 10 \mu \mathrm{g} / \mathrm{ml} \mathrm{leu-}$ peptin and aprotinin, $1 \mathrm{mM}$ phenylmethylsulfonyl fluoride, and $0.5 \mathrm{mM}$ 4-deoxypyridoxine) were incubated with $50 \mu \mathrm{M}$ sphingosine (prepared either in mixed micelles with Triton X-100 or in BSA complexes without Triton X-100), $10 \mu \mathrm{Ci}$ of $\left[\gamma^{-32} \mathrm{P}\right] \mathrm{ATP}(1 \mathrm{mM})$, and $10 \mathrm{mM} \mathrm{MgCl}_{2}$. Labeled lipids were extracted and resolved by TLC as described previously (49). Labeled S1P was quantified with a PhosphorImager (Fujifilm).

Measurement of S1P, dibydroS1P, sphingosine, dibydrosphingosine, ceramide, and sphingomyelin levels. These sphingolipids were measured by high-performance liquid chromatography-tandem mass spectrometry LC-MS at the Lipidomics Core Facility of the Medical University of South Carolina on a Thermo Finnigan TSQ 7000 triple quadrupole mass spectrometer (Thermo Fischer Scientific), operating in a multiple reaction monitoring-positive ionization mode using tissue homogenates corresponding to $1 \mathrm{mg}$ of protein as described (50).
Glycerophospholipid analysis. Uteri were subjected to phospholipid profile analyses (performed by Lipomics). In brief, the technique used involves extraction of tissue lipids with authentic internal standards added by the method described previously (51) using chloroform-methanol (2:1, vol/vol). Samples (40 mg each) were obtained from the whole uteri of nonpregnant mice and the decidua of day 6.5 and 7.5 pc pregnant mice from which the embryos had been removed. Preparative TLC was used to separate individual lipid classes within each extract. Authentic lipid class standard compounds were run on the 2 outside lanes of the same TLC plate to enable identification of the sample lipid classes. Individual lipid fractions were scraped from the plate and transesterified in $3 \mathrm{~N}$ methanolic $\mathrm{HCl}$ in a sealed vial under nitrogen at $100^{\circ} \mathrm{C}$ for 45 minutes. Fatty acid methyl esters obtained were extracted with hexane containing $0.05 \%$ butylated hydroxytoluene and used for gas chromatography after sealing of the hexane extracts under nitrogen. Separation and quantification of fatty acid methyl esters were achieved with capillary gas chromatography, using a gas chromatograph (6890; HP) with a 30-m DB-225MS capillary column (J \& W Scientific), and a flame ionization detector.

PG measurement. Nonpregnant whole uteri and 7.5 pc decidua of pregnant mice, from which the embryos had been removed, were immediately frozen. The uteri were homogenized in $15 \%$ methanol in $0.1 \mathrm{M}$ sodium phosphate buffer ( $\mathrm{pH}$ 7.5). The homogenate was applied to a preconditioned $\mathrm{C}_{18}$ Sep-Pak column (Waters), followed by washing with $15 \%$ methanol and petroleum ether. The PGs were eluted by methyl formate and evaporated under nitrogen gas. The $\mathrm{PGE}_{2}$ levels were determined using the Enzyme Immunoassay for $\mathrm{PGE}_{2}$ (Oxford Biochemical Research) according to the manufacturer's instructions.

Whole-mount in situ bybridization. In situ hybridization was performed essentially as described previously (52). The cDNA fragment corresponding to the entire open reading frame and the fragment including part of the $\mathrm{C}$ terminus and $3^{\prime}$ untranslated region of mouse Sphk1 were used as probes for hybridization. A uterus was cut into 2 halves through an embryo, and one-half was hybridized with the antisense probe while the other was hybridized with the sense probe.

Histological analysis. Ovaries and uteri were fixed in $10 \%$ formalin and processed to be embedded in paraffin. Serial sections $(5 \mu \mathrm{m})$ were made at 10-30 $\mu \mathrm{m}$ intervals and stained with $\mathrm{H} \& \mathrm{E}$ for general morphology. Paraffin sections were deparaffinized and rehydrated. Endogenous peroxidase activity was quenched by incubation with $3 \%$ hydrogen peroxide in water for 5 minutes. Specimens were incubated with rabbit anti-phospho-histone H3 (1:100; 6-570, Millipore), rabbit anti-cytokeratin (1:50; Z0622, Dako), rabbit anti-desmin (1:40; D8241, Sigma-Aldrich), or rat anti-neutrophils (1:50; MCA771GA, AbD Serotec) overnight at $4^{\circ} \mathrm{C}$ to label mitotic cells, trophoblast cells, decidual cells, or neutrophils, respectively. After that, they were reacted with peroxidase-conjugated goat anti-rabbit IgG or goat anti-rat IgG for 30 minutes at room temperature, then visualized with diaminobenzidine/hydrogen peroxide. Cytokeratin antigens were retrieved 
by trypsin incubation for 20 minutes, and desmin antigens were retrieved by Target Retrieval Solution (Dako) at $95^{\circ} \mathrm{C}$ for 20 minutes.

TUNEL assay. The TUNEL assay was performed using the Tdt-FragEL DNA Fragmentation Detection Kit (Calbiochem; EMD Biosciences), according to the manufacturer's instructions.

Statistics. Data are expressed as mean \pm SE. Results having $P$ values of less than 0.05 by the paired Student's $t$ test were considered significant.

\section{Acknowledgments}

We are very grateful to Michael Eckhaus for help with histological examination. This research was supported by the Intramural
Research Program of the NIH, NIDDK, and by NIH Heath grant C06 RR018823.

Received for publication October 17, 2006, and accepted in revised form June 26, 2007.

Address correspondence to: Richard L. Proia, Genetics of Development and Disease Branch, NIDDK, NIH, Bldg. 10, Rm. 9D06, 10 Center Dr., MSC 1821, Bethesda, Maryland 20892-1821, USA. Phone: (301) 496-4391; Fax: (301) 496-0839; E-mail: proia@nih.gov.
1. Dey, S.K., et al. 2004. Molecular cues to implantation. Endocr. Rev. 25:341-373.

2. Wang, H., and Dey, S.K. 2006. Roadmap to embryo implantation: clues from mouse models. Nat. Rev. Genet. 7:185-199.

3. Tan, J., et al. 2002. Evidence for coordinated interaction of cyclin D3 with $\mathrm{p} 21$ and cdk6 in directing the development of uterine stromal cell decidualization and polyploidy during implantation. Mech. Dev. 111:99-113.

4. Zygmunt, M., Herr, F., Munstedt, K., Lang, U., and Liang, O.D. 2003. Angiogenesis and vasculogenesis in pregnancy. Eur. J. Obstet. Gynecol. Reprod. Biol. 110:S10-S18.

5. Olivera, A., and Spiegel, S. 1993. Sphingosine-1phosphate as second messenger in cell proliferation induced by PDGF and FCS mitogens. Nature. 365:557-560.

6. Cuvillier, O., et al. 1996. Suppression of ceramidemediated programmed cell death by sphingosine1-phosphate. Nature. 381:800-803.

7. Olivera, A., et al. 1999. Sphingosine kinase expression increases intracellular sphingosine-1-phosphate and promotes cell growth and survival. J. Cell. Biol. 147:545-558.

8. Spiegel, S., and Milstien, S. 2003. Sphingosine-1phosphate: an enigmatic signaling lipid. Nat. Rev. Mol. Cell Biol. 4:397-407.

9. Lee, M.J., et al. 1999. Vascular endothelial cell adherens junction assembly and morphogenesis induced by sphingosine-1- phosphate. Cell. 99:301-312.

10. Liu, Y., et al. 2000. Edg-1, the G-protein-coupled receptor for sphingosine-1-phosphate, is essential for vascular maturation. J. Clin. Invest. 106:951-961.

11. Kono, M., et al. 2004. The sphingosine-1-phosphate receptors $\mathrm{S}_{1} \mathrm{P}_{1}, \mathrm{~S}_{1} \mathrm{P}_{2}$, and $\mathrm{S}_{1} \mathrm{P}_{3}$ function coordinately during embryonic angiogenesis. J. Biol. Chem. 279:29367-29373.

12. Mizugishi, K., et al. 2005. Essential role for sphingosine kinase in neural and vascular development. Mol. Cell. Biol. 25:11113-11121.

13. Kupperman, E., An, S., Osborne, N., Waldron, S., and Stainier, D.Y. 2000. A sphingosine-1-phosphate receptor regulates cell migration during vertebrate heart development. Nature. 406:192-195.

14. Allende, M.L., Dreier, J.L., Mandala, S., and Proia, R.L. 2004. Expression of the sphingosine-1-phosphate receptor, $\mathrm{S}_{1} \mathrm{P}_{1}$, on $\mathrm{T}$-cells controls thymic emigration. J. Biol. Chem. 279:15396-15401.

15. Matloubian, M., et al. 2004. Lymphocyte egress from thymus and peripheral lymphoid organs is dependent on S1P receptor 1. Nature. 427:355-360.

16. Cinamon, G., et al. 2004. Sphingosine 1-phosphate receptor 1 promotes $B$ cell localization in the splenic marginal zone. Nat. Immunol. 5:713-720.

17. Hannun, Y.A., and Obeid, L.M. 2002. The ceramidecentric universe of lipid-mediated cell regulation: stress encounters of the lipid kind. J. Biol. Chem. 277:25847-25850.

18. Kolesnick, R. 2002. The therapeutic potential of modulating the ceramide/sphingomyelin pathway. J. Clin. Invest. 110:3-8. doi:10.1172/JCI200216127.

19. Kohama, T., et al. 1998. Molecular cloning and functional characterization of murine sphingosine kinase. J. Biol. Chem. 273:23722-23728.

20. Liu, H., et al. 2000. Molecular cloning and functional characterization of a novel mammalian sphingosine kinase type 2 isoform. J. Biol. Chem. 275:19513-19520.

21. Allende, M.L., et al. 2004. Mice deficient in sphingosine kinase 1 are rendered lymphopenic by FTY720. J. Biol. Chem. 279:52487-52492.

22. Zemann, B., et al. 2005. Sphingosine kinase type 2 is essential for lymphodepletion induced by the immunomodulatory drug FTY720. Blood. 107:1454-1458.

23. Adamson, S.L., et al. 2002. Interactions between trophoblast cells and the maternal and fetal circulation in the mouse placenta. Dev. Biol. 250:358-373.

24. Hirashima, M., Lu, Y., Byers, L., and Rossant, J. 2003. Trophoblast expression of fms-like tyrosine kinase 1 is not required for the establishment of the maternal-fetal interface in the mouse placenta. Proc. Natl. Acad. Sci. U. S. A. 100:15637-15642.

25. Oliveira, S.F., Greca, C.P., Abrahamsohn, P.A., Reis, M.G., and Zorn, T.M. 2000. Organization of desmin-containing intermediate filaments during differentiation of mouse decidual cells. Histochem. Cell Biol. 113:319-327.

26. Yoshie, M., Tamura, K., Hara, T., and Kogo, H. 2006. Expression of stathmin family genes in the murine uterus during early pregnancy. Mol. Reprod. Dev. 73:164-172.

27. Pewzner-Jung, Y., Ben-Dor, S., and Futerman, A.H. 2006. When do Lasses (longevity assurance genes) become CerS (ceramide synthases)? Insights into the regulation of ceramide synthesis. J. Biol. Chem. 281:25001-25005.

28. Edsall, L.C., and Spiegel, S. 1999. Enzymatic measurement of sphingosine 1-phosphate. Anal.Biochem. 272:80-86.

29. Lim, H., et al. 1997. Multiple female reproductive failures in cyclooxygenase 2-deficient mice. Cell. 91:197-208.

30. Morita, Y., et al. 2000. Oocyte apoptosis is suppressed by disruption of the acid sphingomyelinase gene or by sphingosine-1-phosphate therapy. Nat. Med. 6:1109-1114.

31. Tilly, J.L., and Kolesnick, R.N. 2003. Realizing the promise of apoptosis-based therapies: separating the living from the clinically undead. Cell Death Differ. 10:493-495.

32. Linzer, D.I., and Fisher, S.J. 1999. The placenta and the prolactin family of hormones: regulation of the physiology of pregnancy. Mol. Endocrinol. 13:837-840.

33. Soares, M.J. 2004. The prolactin and growth hormone families: pregnancy-specific hormones/ cytokines at the maternal-fetal interface. Reprod. Biol. Endocrinol. 2:51.

34. Merrill, A.H., Jr. 2002. De novo sphingolipid biosynthesis: a necessary, but dangerous, pathway. J. Biol. Chem. 277:25843-25846.

35. Nava, V.E., et al. 2000. Sphingosine enhances apoptosis of radiation-resistant prostate cancer cells. Cancer Res. 60:4468-4474.

36. Cuvillier, O., et al. 2001. Sphingosine generation, cytochrome $c$ release, and activation of caspase-7 in doxorubicin-induced apoptosis of MCF7 breast adenocarcinoma cells. Cell Death Differ. 8:162-171.

37. Zhao, Y., et al. 2007. Intracellular generation of sphingosine-1-phosphate in human lung endothelial cells. J. Biol. Chem. 282:14165-14177.

38. Ye, X., et al. 2005. $\mathrm{LPA}_{3}$-mediated lysophosphatidic acid signaling in embryo implantation and spacing. Nature. 435:104-107.

39. Skaznik-Wikiel, M.E., Kaneko-Tarui, T., Kashiwagi, A., and Pru, J.K. 2006. Sphingosine-1-phosphate receptor expression and signaling correlate with uterine prostaglandin-endoperoxide synthase 2 expression and angiogenesis during early pregnancy. Biol. Reprod. 74:569-576.

40. Sugi, T., and McIntyre, J.A. 1995. Autoantibodies to phosphatidylethanolamine $(\mathrm{PE})$ recognizes a kininogen-PE complex. Blood. 86:3083-3089.

41. Sugi, T., and McIntyre, J.A. 2001. Certain autoantibodies to phosphatidylethanolamine $(\mathrm{aPE})$ recognize factor XI and prekallikrein independently or in addition to the kininogens. J. Autoimmun. 17:207-214.

42. Adam, A., Damas, J., Galay, G., and Bourdon, V. 1989. Quantification of rat T-kininogen using immunological methods. Biochem. Pharmacol. 38:1569-1575.

43. Brann, D.W., Greenbaum, L., Mahesh, V.B., and Gao, X. 1995. Changes in kininogens and kallikrein in the plasma, brain, and uterus during pregnancy in the rat. Endocrinology. 136:46-51.

44. Hossain, A.M., Whitman, G.F., and Khan, I. 1995. Kininogen present in rat reproductive tissues is apparently synthesized by the liver, not by the reproductive system. Am. J. Obstet. Gynecol. 173:830-834.

45. Hermann, A., Buchinger, P., Somlev, B., and Rehbock, J. 1996. High and low molecular weight kininogen and plasma prekallikrein/plasma kallikrein in villous capillaries of human term placenta. Placenta. 17:223-230.

46. Tian, Y., et al. 2006. Placental thrombosis and spontaneous fetal death in mice deficient in ethanolamine kinase 2. J. Biol. Chem. 281:28438-28449.

47. Johnstone, E.D., et al. 2005. Sphingosine-1-phosphate inhibition of placental trophoblast differentiation through a $\mathrm{G}_{\mathrm{i}}$-coupled receptor response. J. Lipid Res. 46:1833-1839.

48. Hudson, N.K., et al. 2007. Modulation of human arterial tone during pregnancy: the effect of the bioactive metabolite sphingosine-1-phosphate. Biol. Reprod. 77:45-52.

49. Olivera, A., Kohama, T., Tu, Z., Milstien, S., and Spiegel, S. 1998. Purification and characterization of rat kidney sphingosine kinase. J. Biol. Chem. 273:12576-12583.

50. Bielawski, J., Szulc, Z.M., Hannun, Y.A., and Bielawska, A. 2006. Simultaneous quantitative analysis of bioactive sphingolipids by high-performance liquid chromatography-tandem mass spectrometry. Methods. 39:82-91.

51. Folch, J., Lees, M., and Sloane-Stanley, G.H. 1957. A simple method for the isolation and purification of total lipids from animal tissues. J. Biol. Chem. 226:497-509.

52. Wilkinson, D.G. 1992. In situ bybridization: a practical approach. IRL Press. Oxford, United Kingdom. 163 pp. 